\title{
Die Rechtslage beim bundesweiten Stadionverbot
}

\author{
Jan F. Orth/Björn Schiffbauer*
}

A. Die besonderen Eigenschaften des Mittels „Stadionverbot“" 179

I. Das Stadionverbot als privatrechtliche Präventivmaßnahme........... 179

II. Das Stadionverbot als Durchbrechung eines Kontrahierungszwangs.... 179

B. Stadionverbotsrichtlinien und strafrechtlicher Einfluss ................. 181

I. Anwendungsbereich der SVRL ..... 182

1. Sachlich ....................... 182

2. Örtlich ....................... 182

3. Persönlich.................... 183

II. Rechtsnatur und Funktionsmechanismen der SVRL .................. 184

1. Rechtsnatur................... 184

2. Funktionsmechanismen......... 185

III. Inhalt und Wirkung der SVRL ..... 186

1. Kategorien und Festsetzungsmöglichkeiten eines Stadionverbots ......................... 186

a) Festsetzung mit und ohne Ermessen................. 187

b) Örtliches und überörtliches Stadionverbot ............... 188

c) Verdachtsbegründetes und verstoßbegründetes Stadionverbot....

2. Dauer und Aufhebung eines Stadionverbots 189

a) Grundsätzliche zeitliche Grenzen .... 189

b) Reduzierung, Aussetzung, Aufhebung ................. 190

3. Übrige Bestimmungen der SVRL

IV. Kritik

1. Zweifelhafte Rechtsverbindlichkeit ........................ 192

2. Die Einleitung eines Ermittlungsverfahrens als Grundlage........

3. Die Art der Verfahrenseinstellung als Vorgabe für die Aufhebung eines Stadionverbots.... a) Staatsanwaltliche Einstellungspraxis ................. 194

b) Fehlende Rechtsmittel....... 194

c) Zwischenergebnis ........... 195

4. Weitere Defizite der SVRL ....... 195

C. Zivilrechtliche Grundlagen........... 196

I. Anspruch aus $\mathbb{S} \mathbb{S} 1004$ Abs. 1 Satz 2

(analog), 823 Abs. 1 BGB........... 196

1. Beeinträchtigung von Eigentum oder einem sonstigen „absoluten Recht" (petitorisch)........... 196

2. Geltendmachung im fremden Namen ......................... 197

3. Keine Duldungspflicht, $\mathbb{S} 1004$ Abs. 2 BGB ..................... 198

4. Wiederholungsgefahr............ 198

a) Herrschende Meinung....... 198

b) Meinungen zur Gefahrprognose für das Stadionverbot..................... 198

c) Auffassung des BGH zur Gefahrprognose beim Stadionverbot....

aa) Objektive Grundlagen einer „Zugehörigkeit“............ 202

bb) Subjektive Einflüsse auf Zugehörigkeit.............. 203

cc) Rechtsfolgen einer Zugehörigkeit..................... 203

d) Zwischenergebnis ........... 204

II. Anspruch aus $\$ 862$ Abs. 1 Satz 2 BGB ............................ 204

1. Beeinträchtigung von „Besitz“ (possessorisch) .................. 204

2. Wiederholungsgefahr............ 204

3. Verbotene Eigenmacht.......... 205

4. Konkurrenzen ................. 205

5. Grenzen, insbesondere $\mathbb{\$} 864$

BGB ......................... 205

6. Zwischenergebnis ............... 205

III. Nebenansprüche aus Besitzstörung .............................. 206

* Der Autor Orth ist stellvertretender Vorsitzender eine großen Strafkammer am LG Köln, Lehrbeauftragter für Sportrecht an der Universität zu Köln und Präsidiumsmitglied des Fußball-Verbandes Mittelrhein (FVM). Der Autor Schiffbauer ist Rechtsreferendar beim LG Köln, Wiss. Mitarbeiter am Lehrstuhl für deutsches Strafrecht, europäisches Strafrecht, Völkerstrafrecht sowie Friedenssicherungs- und Konfliktsvölkerrecht von Prof. Dr. Claus Kreß LL.M. (Cambridge) der Universität zu Köln und stellvertretender Vorsitzender des Rechts- und Satzungsausschusses des FVM. 
D. Einfluss des Verfassungsrechts....... 206

I. Wirkung von Grundrechten in rein privatrechtlichen Beziehungen....... 206

II. Grundrechtsbeeinträchtigungen in concreto........................ 207

1. Art. 11 Abs. 1 GG - Freizügigkeit.......................... 207

2. Art. 2 Abs. 1 i.V.m. Art. 1 Abs. 1 GG - Allgemeines

Persönlichkeitsrecht............ 208

a) Die praxisübliche Ausübung des Hausrechts als geeignetes Mittel...................... 209

b) Das Stadionverbot in der Mittel-Zweck-Relation ...... 210

3. Art. 3 Abs. 1 GG - Willkürverbot. a) Stadionverbotsfestsetzung und bestätigende Entscheidung direkt................ 211

b) Fortsetzung staatlicher Willkür

III. Verfassungsrechtliche Dimensionen der BGH-Entscheidung zum Stadionverbot

1. Spezifische Grundrechtsverstöße

2. Fehlerhafte Rechtsanwendung durch den BGH ....

E. Lösungsvorschlag: Ein vorläufiges Sta-

dionverbot....

F. Schlussbemerkungen ................. 217

„Fußballfans sind keine Verbrecher" 1 - oder doch?² Das ist die pragmatisch-polemische Zusammenfassung der in den Medien und durch die Rechtsprechung ${ }^{3}$ aufgerufenen Frage nach der Rechtmäßigkeit von bundesweiten Stadionverboten auf Grundlage einer Gefahrenprognose, der möglicherweise nur geringe tatsächliche Erkenntnisse zu Grunde liegen. Juristisch lässt sie sich nur dann abschließend und zuverlässig beantworten, wenn man hierzu sämtliche Implikationen zivil-, öffentlichund strafrechtlicher Natur heranzieht. Erstmals hat sich auch der BGH zu bundesweiten Stadionverboten geäußert. ${ }^{4}$ Die Entscheidung enthielt vordergründig wenig Neues. Die bisherigen wissenschaftlichen Reaktionen ${ }^{5}$ auf das Urteil beschränkten sich auf Teilaspekte; eine rechtswissenschaftliche Durchleuchtung des Präventionsmittels „Stadionverbot“ als Gesamtbetrachtung unterblieb bislang. ${ }^{6}$ Die Autoren unternehmen mit diesem Beitrag den Versuch, diese Lücke zu schließen und gehen dabei insbesondere der Frage nach, ob das Thema auch unter verfassungsrechtlichen

1 Bekannter Schlachtruf deutscher Fußballfans im Stadion, wenn ein dort durchgeführter Polizeieinsatz als überhöht empfunden wird.

2 Den Autoren liegt daran festzuhalten, dass sie sich von jeder Form von Gewalt im Zusammenhang mit Fußballspielen distanzieren und auch die präventive Maßnahme des Stadionverbots grundsätzlich für sinnvoll und richtig erachten. Die Ausführungen sollen einen Beitrag zu noch größerer Einzelfallgerechtigkeit leisten.

3 Der jüngst für Aufsehen sorgende Fall entsprang am AG Duisburg (unveröffentlicht) mit erfolgloser Berufung beim LG Duisburg, SpuRt 2009, 78.

4 BGH, Urt. v. 30.10.2009, Az. V ZR 253/08, NJW 2010, 534 = SpuRt 2010, 28.

5 Breucker, Anmerkung, SpuRt 2010, 31; Heermann, Anmerkung, NJW 2010, 537; Klesczewski, Anmerkung, JZ 2010, 251 ff.; Walker/Klopp, Anmerkung, LMK 2010, 295984.

6 Vor der BGH-Entscheidung äußerten sich: Breucker, Zulässigkeit von Stadionverboten, JR 2005, 133 ff.; Orth, Vereins- und Verbandsstrafen am Beispiel des Fußballsports, Frankfurt 2009, S. 135 ff. 
Aspekten ${ }^{7}$ ausreichend untersucht ist. Dies ist schon alleine deswegen notwendig, weil eine Verfassungsbeschwerde gegen die BGH-Entscheidung eingelegt wurde. ${ }^{8}$

\section{A. Die besonderen Eigenschaften des Mittels „Stadionverbot“}

Der privatrechtliche Einsatz eines Mittels, wie es das Stadionverbot verkörpert, ist in der deutschen Rechtslandschaft einzigartig: In keinem anderen Lebensbereich ist es üblich, ein Hausverbot gleichsam automatisch auch im Namen anderer Hausrechtsinhaber auf unterschiedliche Orte auszudehnen. Daher ist es für einen ersten Schritt unerlässlich, die besonderen Eigenschaften dieser Maßnahme zu vergegenwärtigen, um vor diesem Hintergrund in die eigentliche Analyse einzusteigen.

\section{Das Stadionverbot als privatrechtliche Präventivmaßnahme}

Beim Stadionverbot handelt es sich um eine auf Grundlage des Zivilrechts ergehende Präventivmaßnahme, mit der dem Betroffenen für einen gewissen Zeitraum untersagt wird, ein Fußballstadion zu betreten, damit zukünftig von ihm keine weiteren sicherheitsrelevanten Beeinträchtigungen im Zusammenhang mit Fußballspielen ausgehen können. Es handelt sich um eine auf dem Hausrecht der jeweiligen Stadioninhaber beruhende vorbeugende Maßnahme, nicht etwa um eine seitens der beteiligten Vereine oder Verbände ausgesprochene Vereins- oder Verbandsstrafe. ${ }^{9}$ Dass gerade das bundesweite Stadionverbot - der Ausschluss von sämtlichen überregional organisierten Fußballspielen regelmäßig für mehrere Jahre - als Reaktion auf Gewaltvorfälle um Fußballspiele von den Fans gleichwohl als Sanktion empfunden wird, liegt auf der Hand. ${ }^{10}$ Derzeit sind etwa 3.500 Personen von einem Stadionverbot betroffen; die Tendenz ist steigend. ${ }^{11}$

\section{Das Stadionverbot als Durchbrechung eines Kontrahierungszwangs}

Es überrascht zunächst, dass dieses Rechtsinstitut derart rege Diskussionen und kaum überschaubare Rechtsfragen auslösen soll. Denn ein Fußballspielveranstalter, der über das von ihm genutzte Stadion das Hausrecht ausüben kann und gegenüber einem Stadionbesucher ein Stadionverbot ausspricht, braucht sich im Ausgangspunkt nicht dafür zu rechtfertigen. Etwas Anderes kann nur gelten, wenn der Veranstalter nicht grundsätzlich frei entscheiden kann, welchen Zuschauer er in das von

7 Nicht behandelt werden die gleichermaßen umstrittenen sportpolitischen Aspekte, insbesondere im angespannten Verhältnis zwischen Polizeiverwaltung und Fanorganisation. Lesenswert: „Fußball und Gewalt" von Köster im Magazin 11Freunde, Ausgabe Nr. 98, Januar 2010, S. 114 ff.

8 Spiegel online vom 5. Dezember 2009, http://www.spiegel.de/sport/fussball/0,1518,665378,00.html. Anhängig unter dem Az. 1 BvR 3080/09 beim 1. Senat des BVerfG.

9 Dies scheidet schon deswegen aus, weil der bloße Stadionbesucher nicht Mitglied der beteiligten Vereine oder gar mittelbares Mitglied ist, sodass er deren Strafgewalt nicht unterliegt. Auch durch AGB auf Eintrittskarten kann sich der Zuschauer dieser Strafgewalt nicht wirksam unterwerfen, vgl. Orth, a.a.O. (s.o. Fn. 6), S. 128 (II.), 146 (II.) und $200 \mathrm{ff}$.

10 Orth, a.a.O. (s.o. Fn. 6), S. 135 m.w.N.

11 http://www.tagesschau.de/inland/fangewalt102.html. 
ihm genutzte Stadion hineinlässt. Dies betrifft also das Zustandekommen eines Stadionbenutzungsvertrags zwischen Veranstalter und Fan, der sich ausschließlich auf privatrechtlicher Ebene abspielt. Der Stadionbetreiber müsste also einem zivilrechtlichen Kontrahierungszwang ${ }^{12}$ unterliegen. Dieser ergibt sich im Grundsatz aus dem Deliktsrecht, wenn die Ablehnung eines Vertragsschlusses eine unerlaubte Handlung darstellt. ${ }^{13}$ Denn mit der Rechtsfolge der Naturalrestitution nach $\$ 249$ S. 1 BGB ist der hieraus resultierende Schadenersatzanspruch auf den Vertragsabschluss gerichtet. ${ }^{14}$ Diese Grundsätze wurden von der Rechtsprechung für $\$ 826$ BGB als grundrechtsrelevante Generalklausel ${ }^{15}$ entwickelt. ${ }^{16}$ Kontrahierungszwang liegt nach herkömmlicher Auffassung also vor, wenn die Ablehnung des Vertragschlusses eine vorsätzliche sittenwidrige Schädigung darstellt. Andererseits ist für Verbraucher dieser Schutz nicht auf die Voraussetzungen des $\mathbb{\$} 826$ BGB beschränkt worden. Vielmehr hat sich ein allgemeiner Rechtssatz entwickelt, nachdem derjenige Unternehmer, der lebenswichtige Güter öffentlich anbietet, den Vertragsschluss nur aus sachlichen Gründen ablehnen darf, sofern für den Kunden keine zumutbare Möglichkeit besteht, seinen Bedarf anderweitig zu decken. ${ }^{17}$ Ein Rekurs auf diese Formel löst die - geführte ${ }^{18}$ - Diskussion aus, ob Fußballspiele „lebenswichtige Güter" darstellen (für Theater, Museen und städtische Badeanstalten soll dies gelten) ${ }^{19}$ oder ob gar die medialen Informationsmöglichkeiten über Fußballspiele den Stadionbesuch ausreichend substituieren können. Unabhängig von dieser wenig zielführenden ${ }^{20}$ Diskussion ist es jedenfalls a priori nicht ausgeschlossen, für Veranstalter von Profifußballspielen einen Kontrahierungszwang über das Deliktsrecht anzunehmen. ${ }^{21}$

Ein solcher ist für Fußballspiele der Bundesligen stets bestritten worden. ${ }^{22}$ Dies dürfte nicht zu halten sein, ${ }^{23}$ insbesondere nicht nach der zitierten BGH-Entscheidung: Wenn der $\mathrm{BGH}^{24}$ ausführt, dass der Veranstalter einen Zuschauer nicht willkürlich, sondern nur mit sachlichem Grund ausschließen darf, weil der Veranstalter die mittelbar in das Zivilrecht einwirkenden Grundrechte des Zuschauers (Allgemeines Per-

12 Vgl. zum Begriff Busche, Privatautonomie und Kontrahierungszwang, Tübingen 1999, S. 110.

13 Palandt-Ellenberger, 69. Aufl. 2010, Einf v \$145, Rn. 9.

14 Busche, a.a.O. (s.o. Fn. 12), S. 151. a.a.O.

15 S. zu den verfassungsrechtlichen Dimensionen des Stadionverbots unten unter D.

16 Palandt-Ellenberger, a.a.O. (s.o. Fn. 13).

17 Staudinger-Bork, BGB (Neubearbeitung 2003), Vorbem. \$\$ 145-156, Rn. 22 f.; Palandt-Ellenberger, Einf v $\$ 145$, Rn. 10.

18 Orth, a.a.O. (s.o. Fn. 6), S. 138 m.w.N.

19 Staudinger-Bork, a.a.O.; Palandt-Ellenberger, a.a.O. (s.o. Fn. 17).

20 Vgl. hierzu ausführlich Busche, a.a.O. (s.o. Fn. 12), S. 200, der die Merkmale des „Normalbedarfs“ oder des „lebensnotwendigen Bedürfnisses“ u.a. wegen der verursachten Rechtsunsicherheit verwirft.

21 Vgl. hierzu Busche, a.a.O. (s.o. Fn. 12), S. 187.

22 AG Frankfurt, Az. 30 C 1600/04-47, Urt. v. 8.10.2004 (unveröffentlicht); LG Duisburg, Az. 7 S 63/05, Urt. v. 22.7.2005 (juris); Breucker, a.a.O. (s.o. Fn. 6), 136.

23 So schon Orth, S. 138 m.w.N.

24 BGH, a.a.O. (s.o. Fn. 4), Rn. 13. 
sönlichkeitsrecht, Gebot der Gleichbehandlung) zu beachten hat, statuiert er nichts Anderes als einen Kontrahierungszwang. Denn ein ohne sachlichen Grund ausgeschlossener Zuschauer könnte nach BGH aus $\mathbb{\$} \$ 826,242$ BGB und nach neuerer Auffassung aus $\mathbb{S} \$ 823$ Abs. 1 BGB i.V.m. Art. 2 Abs. 1 GG, 249 BGB auf Abschluss eines entsprechenden Vertrags klagen. Letztere Auffassung legt dar, dass sich über einen Eingriff in das Allgemeine Persönlichkeitsrecht ein Kontrahierungszwang aus dem Deliktsrecht über $\mathbb{8} 823$ Abs. 1 BGB ergeben kann, wenn das Unterlassen des Vertragsschlusses das Allgemeine Persönlichkeitsrecht des Interessenten ${ }^{25}$ verletzt und es sich um eine sog. „qualifizierte Vertragsverweigerung “ handelt. ${ }^{26}$ Eine solche liegt vor, wenn als zusätzliche Voraussetzungen diejenigen eines allgemeinen Kontrahierungszwangs, nämlich die Verfolgung eines rechtlich geschützten Interesses seitens des Vertragsinteressenten, die Abhängigkeit des Vertragsinteressenten von einem bestimmten Anbieter, die generelle Vertragsgeneigtheit des Anbieters und die Leistungsfähigkeit des Anbieters, hinzukommen. ${ }^{27}$ Beim Stadionbesuch ist alleine fraglich, ob ein „rechtlich geschütztes Interesse“ des Vertragsinteressenten, also des Stadionbesuchers, vorliegt; dies kann aber nach der insoweit eindeutigen BGH-Entscheidung nicht mehr ernsthaft in Frage gestellt werden.

So ist aber auch ein rechtmäßig erteiltes Stadionverbot immer ein sachlicher Grund mit einem Zuschauer nicht kontrahieren zu müssen, also eine Ausnahme vom Kontrahierungszwang. Natürlich dürfen etwa kommunale Grundversorger (Gas, Wasser, Strom), die nach allgemeiner Auffassung einem Kontrahierungszwang unterliegen, ihre Leistungen einstellen oder von einem Vorschuss abhängig machen, wenn der Verbraucher seine vertragliche Gegenleistung (Zahlung des entsprechenden Entgelts) nicht erbringt, vgl. etwa nur $\mathbb{1} 19$ StromGVV. Busche würde einen Kontrahierungszwang in diesem Fall unter dem Tatbestandsmerkmal des ,geschützten rechtlichen Interesses" verneinen. Er sieht vertragskonformes Verhalten des Vertragsinteressenten als Voraussetzung dafür an, ein rechtliches Interesse von der Rechtsordnung als schützenswert anzuerkennen. ${ }^{28}$ Ein randalierender Stadionbesucher verhält sich gerade nicht vertragskonform, sondern verletzt vertragliche Nebenpflichten. ${ }^{29}$

\section{B. Stadionverbotsrichtlinien und strafrechtlicher Einfluss}

Für einen einheitlichen Umgang mit der Maßnahme „Stadionverbot“ erließ der DFB sog. „Richtlinien zur einheitlichen Behandlung von Stadionverboten“ (im Folgenden:

25 Ein zu Unrecht ausgesprochenes Stadionverbot verletzt den Fan in seinem Allgemeinen Persönlichkeitsrecht, s.u. unter D. II. 2.

26 Busche, a.a.O. (s.o. Fn. 12), S. 212, 215.

27 Busche, a.a.O. (s.o. Fn. 12), S. 198.

28 Busche, a.a.O. (s.o. Fn. 12), S. 202.

29 OLG Rostock, Urt. v. 28.4.2006, Az. 3 U 106/05, NJW 2006, 1819. 
Stadionverbotsrichtlinien - SVRL). ${ }^{30}$ Die letzte Fassung gilt seit dem 27.11.2009, jedoch existieren SVRL schon seit dem 1.7.1993 und wurden im Laufe der Zeit durch den $\mathrm{DFB}^{31}$ - zum Teil wesentlich - inhaltlich überarbeitet. ${ }^{32}$

\section{Anwendungsbereich der SVRL}

Die SVRL befassen sich ausschließlich mit Fragen der Voraussetzungen für die Verhängung von Stadionverboten, ihren Rahmenbedingungen und den diesbezüglichen Abwicklungsdetails. Definiert wird das Stadionverbot als

„die auf der Basis des Hausrechts gegen eine natürliche Person wegen sicherheitsbeeinträchtigenden Auftretens im Zusammenhang mit dem Fußballsport, insbesondere anlässlich einer Fußballveranstaltung, innerhalb oder außerhalb einer Platz-oder Hallenanlage vor, während oder nach der Fußballveranstaltung festgesetzte Untersagung bei vergleichbaren zukünftigen Veranstaltungen eine Platz- oder Hallenanlage zu betreten bzw. sich dort aufzubalten" $(\mathbb{S} 1$ Abs. 1 SVRL).

Hieraus lässt sich in Verbindung mit den restlichen Teilen der SVRL ihr Anwendungsbereich klar eingrenzen.

\section{Sachlich}

Die SVRL erklären sich sachlich bei sämtlichen sicherheitsbeeinträchtigenden Situationen im Zusammenhang mit dem Fußballsport für anwendbar. Das Merkmal der Sicherheitsbeeinträchtigung wird durch sie selbst mit Inhalt gefüllt ${ }^{33}$ und emanzipiert sich so von dem grundsätzlichen Anwendungsbereich. Damit erstreckt sich der sachliche Anwendungsbereich auf alle Betätigungsfelder des DFB.

\section{2. Örtlich}

Ein Stadionverbot kann sich lediglich auf das Betreten einer einzigen Sportstätte beziehen (örtliches Stadionverbot) oder aber auf sämtliche Spielorte im Verbandsgebiet des DFB (überörtliches Stadionverbot); ${ }^{34}$ es ist also grundsätzlich national ausgerichtet. Stadionverbote für Fußballspiele im Ausland werden in Deutschland schon wegen mangelnder Zuständigkeit nicht verhängt; doch ist nicht auszuschließen, dass entsprechende Maßnahmen von ausländischen Verbänden oder Vereinen ausgesprochen werden. Im Gegenzug behält sich auch der DFB durch $\mathbb{} 4$ Abs. 5 i.V.m. $\mathbb{} 3$ Abs. 1 Nr. 3 vierter Spiegelstrich SVRL vor, bei als problematisch eingestuftem Fan-

30 Die aktuelle Version kann auf der DFB-Homepage heruntergeladen werden: http://www.dfb.de/ uploads/media/Richtlinien-Stadionverbote-27-11-09.pdf.

31 Grundlegend zur Organisation des deutschen Fußballs: Orth, a.a.O. (s.o. Fn. 6), S. 58 ff.

32 Insbesondere die Tatbestandsvoraussetzungen für die Verhängung eines Stadionverbots unterliefen einen gewissen Wandel, vgl. dazu eingehender unten Fn. 116.

33 S. näher unten unter B. III.

34 S. dazu näher unten unter B. III. b). 
verhalten im Ausland ein bundesweites Stadionverbot festzusetzen. ${ }^{35}$ Damit ist nur der Wirkungsbereich des Stadionverbots national, der Anknüpfungsbereich dagegen international.

\section{Persönlich}

In persönlicher Hinsicht ist zu unterscheiden zwischen den Festsetzungsberechtigten eines Stadionverbots und den durch die Festsetzung belasteten Adressaten. Letztere können nach $\mathbb{S} 1$ Abs. 1 i.V.m. $\mathbb{S} 4$ Abs. 1 SVRL jede natürliche Person sein, ohne dass sie besondere Eigenschaften erfüllen müssten. Insbesondere sind mögliche Vereinszugehörigkeiten ${ }^{36}$ der Betroffenen ohne Bedeutung.

Interessanter gestaltet sich der Kreis der Festsetzungsberechtigten ${ }^{37}$ eines Stadionverbots, die zugleich Adressaten der SVRL sind. Grundsätzlich ist nämlich nur der Eigentümer oder Besitzer der Platz- oder Hallenanlage - im Folgenden kurz „Stadionbetreiber"38 genannt - als „originärer Hausrechtsinhaber“ ( $\mathbb{2}$ Abs. 1 SVRL) angesprochen. Sollte dies einmal nicht ein Verein, ${ }^{39}$ der DFB oder der Ligaverband ${ }^{40}$ sein, ${ }^{41}$ ordnet $\$ 2$ Abs. 2 SVRL an, dass das Hausrecht vom originären Hausrechtsinhaber auf eine der genannten drei Parteien übertragen werden muss; ${ }^{42}$ tatsächlich sind also nur Vereine/Gesellschaften, DFB und Ligaverband festsetzungsberechtigt.

Dies bestätigt und konkretisiert $\ 3$ SVRL, der in abgestufter Priorität den Vereinen (Nrn. 1 und 2), dem DFB (Nr. 3) und dem Ligaverband (Nr. 4) die Zuständigkeit zur Aussprache eines Stadionverbots überträgt. Der Heimverein gilt dabei als vorrangig festsetzungsberechtigt, soweit sich die dem Stadionverbot zu Grunde liegenden

35 So kündigte z.B. der 1. FC Köln während der Vorbereitung zur Saison 2010/2011 an, jeweils ein bundesweites Stadionverbot gegen diejenigen Anhänger zu erwirken, welche im Rahmen einer Fanreise zum Mannschaftstrainingslager in Österreich dort in einer Kneipe randalierten, vgl. http://www.ksta.de/html/artikel/1279878032520.shtml.

36 Dieses Kriterium ist in anderen sportrechtlichen Zusammenhängen dagegen von entscheidender Bedeutung, s. dazu Nachweise in Fn. 9

37 Das hier der Einfachheit halber als „Festsetzungsberechtigung“ Bezeichnete gilt entsprechend auch auf Rechtsfolgenseite für Reduzierung, Aussetzung und Aufhebung eines Stadionverbots (dazu mehr unten unter B. III. 2.). Auch wenn $\mathbb{3}$ Abs. 1 SVRL etwas missverständlich die Vokabel „obliegt“ verwendet, handelt es sich dennoch um eine Berechtigung, soweit nicht über den bloßen Anwendungsbereich hinaus die SVRL das Ermessen des Festsetzungsberechtigten auf Null reduzieren; Näheres zum Ermessen unten unter B. III. 1.

$38 \mathrm{Zu}$ den im professionellen Fußballsport üblichen Konstellationen der Sportstättenbetreibung vgl. instruktiv Orth, a.a.O. (s.o. Fn. 6), S. 136.

39 Gemeint sind entsprechend dem Wirkungskreis des DFB und nach der Präambel der SVRL die „Vereine und Kapitalgesellschaften („Tochtergesellschaften“) der Lizenzligen, der 3. Liga und der Regionalligen“, die im Folgenden der Einfachheit halber pauschal als „Verein“ bezeichnet werden sollen.

40 Zur Struktur im Deutschen Fußball - insbesondere die Zuständigkeitsaufteilung zwischen DFB und Ligaverband - vgl. Orth, s.o. Fn. 31.

41 Denkbar wäre z.B. der praxisübliche Fall, dass das Stadion im Eigentum einer GmbH als Betreibergesellschaft steht, die dann ihr aus dem Eigentum abgeleitetes Hausrecht auf den benutzenden Verein überträgt.

42 Dies kann eigentlich nur auf die seltenen Fälle beschränkt sein, in denen DFB, Ligaverband oder Vereine/Gesellschaften Veranstalter in einem Stadion, gleichzeitig aber nicht Besitzer des Stadions im zivilrechtlichen Sinne sind. 
Handlungen entweder auf seiner Platzanlage (Nr. 1 dritter Spiegelstrich) oder innerhalb der Kommune seines Vereinssitzes (Nr. 1 vierter Spiegelstrich) ereignet haben. Erst wenn diese Tatbestandsvoraussetzungen nicht erfüllt sind, steht auch „dem Verein, der eine Reise zu einer Fußballveranstaltung organisiert und betreut“ (Nr. 2) - also dem Gastverein - eine Festsetzungsberechtigung zu. Diese erstreckt sich allerdings wohl nur auf Bezugstatsachen, welche sich während der beschriebenen, organisierten und betreuten "Reise“ ereignen. ${ }^{43}$

Hilfsweise erlangt auch der DFB - wenn er nicht selbst Veranstalter eines Fußballspiels ist ${ }^{44}$ oder eine Auslandshandlung vorliegt ${ }^{45}$ - eine Festsetzungsberechtigung, „soweit die Zuständigkeit eines Vereins nicht gegeben ist“ (Nr. 3 dritter Spiegelstrich). Wiederum hilfsweise gilt dies bei gleichem Wortlaut auch für den Ligaverband (Nr. 4 zweiter Spiegelstrich). Diese vermeintlichen Auffangkompetenzen gelten zwar grundsätzlich nur subsidiär; wenn sie aber einmal zur Anwendung kommen, unterliegen sie dagegen nicht denjenigen Grenzen, welche für Vereine gelten.

\section{Rechtsnatur und Funktionsmechanismen der SVRL}

Nach Klarstellung des Anwendungsbereichs soll in einem nächsten Schritt auf die Rechtsnatur und Funktionsmechanismen der SVRL eingegangen werden.

\section{Rechtsnatur}

Bei den SVRL handelt es sich um verbandliches Innenrecht. Sie werden auf Grund der Ermächtigungsgrundlage des $\$ 31$ Abs. 2 der Richtlinien des DFB zur Verbesserung der Sicherheit bei Bundesspielen (RVSB, eines von sechs als „Durchführungsbestimmungen “ bezeichneten Regelungswerken) ${ }^{46}$ von der DFB-Kommission für Prävention und Sicherheit erlassen. Durchführungsbestimmungen kann gemäß $\$ 51$ SpO/DFB der DFB-Spielausschuss mit Zustimmung des DFB-Präsidiums erlassen. Die SpO/DFB selbst ist gemäß $\$ \$ 6$ Nr. 2 lit. a) und Nr. 4, 24 Nr. 2 lit. f) DFB-Satzung vom DFB-Bundestag beschlossen worden. Auf Grund der doppelten Delegation, zudem aus einer "Ordnung" heraus und nicht aus der DFB-Satzung, stehen die SVRL in dritter Ebene unter dem Satzungsrecht. Bei den SVRL handelt es sich daher um eine „Ordnung“ im vereinsrechtlichen Sinne, weil sie ein verbandlicher Rechtssatz unterhalb der Satzungsebene sind. ${ }^{47}$ Damit kommt ihnen im staatlichen Recht,

43 Die SVRL schweigen zu den genauen Voraussetzungen, doch lässt der Richtlinienkontext keinen anderen Schluss zu, ohne dass dem Gastverein entweder eine umfassende oder aber eine auf Null reduzierte Festsetzungsberechtigung eröffnet wird.

44 Dazu gehört auch, aber nicht nur das in den SVRL separat aufgeführte DFB-Pokalfinale.

45 S. oben unter B. I. 2.

46 Die sechs Durchführungsbestimmungen zur SpO/DFB: Allgemeinverbindliche Vorschriften über die Beschaffung und Ausgestaltung der Spielkleidung, Anti-Doping-Richtlinien, RVSB, Fußballspiele in der Halle, Richtlinien für Benefiz- und Abschiedsspiele und Richtlinien für Spiele mit ausländischen Mannschaften.

47 Reichert, Vereins- und Verbandsrecht, 12. Aufl., Köln 2010, Rn. 470; Summerer, in: Fritzweiler/Pfister/Summerer (Hrsg.), Praxishandbuch Sportrecht, München 2007, Rn. 2/8. 
insbesondere im Polizei- und Ordnungsrecht, naturgemäß erst einmal keine eigenständige Wirkung zu.

\section{Funktionsmechanismen}

Die SVRL sollen das Aussprechen von bundesweiten und örtlichen Stadionverboten ermöglichen. Für ein örtliches Stadionverbot sind sie, abgesehen von Gleichbehandlungsaspekten, wenig hilfreich, denn selbstverständlich kann ein Stadionbetreiber für „sein“ Stadion beim Vorliegen einer entsprechenden Gefahrprognose (s.u. unter C. I. 4.) immer auf Grundlage einer individuellen einzelfallbezogenen Beurteilung ein örtliches Stadionverbot auch ohne Bezugnahme auf die SVRL verhängen. Wie gesagt sind die SVRL insoweit nur für eine bundesweit einheitliche Handhabung sinnvoll; konstitutiver Charakter kommt ihnen nicht zu.

Adressaten der SVRL sind die Festsetzungsberechtigten (s.o. unter B. I. 3.), also die Vereine und Kapitalgesellschaften der Bundesligen (Bundesliga, 2. Bundesliga, 3. Liga) und der Regionalligen sowie der DFB und der Ligaverband (vgl. Präambel der SVRL).

Damit schließlich bundesweite Stadionverbote möglich werden, sieht $\mathbb{} 1 \mathrm{Abs} .5$ SVRL vor, dass sich alle Festsetzungsberechtigten gegenseitig zur Ausübung des Hausrechts auch für das Stadion, über welches der jeweilige Stadionbetreiber sein Hausrecht hat, bevollmächtigen. In der entsprechenden Mustervorlage für die Vereine und Kapitalgesellschaften heißt es u.a.:

2. Der [Verein] ist damit einverstanden, dass der DFB, der Ligaverband sowie die jeweiligen Vereine und Gesellschaften der Bundesliga, der 2. Bundesliga, der 3. Liga und der Regionalligen auf der Grundlage der Sicherheitsrichtlinien ein auch für seine Platzanlage geltendes, bundesweit wirksames Stadionverbot aussprechen, reduzieren, aussetzen und/oder aufheben können. Die erforderlichen Ermächtigungen und Vollmachten werden hiermit erteilt. Der DFB wird dies den betreffenden Vereinen und Gesellschaften mitteilen. Bereits erlassene Stadionverbote werden übernommen und als verbindlich anerkannt.

3. Der [Verein] verpflichtet sich, bei Vorliegen der in den Sicherheitsrichtlinien festgelegten Voraussetzungen ebenfalls ein bundesweit wirksames Stadionverbot auszusprechen. [...]

5. Die in diesen Erklärungen enthaltenen Ermächtigungen und Verpflichtungen gelten gegenseitig und obne Einschränkung.

Seit einer Entscheidung des AG Frankfurt ${ }^{48}$ sind wegen der Zurückweisungsmöglichkeit des $\mathbb{S} 174$ BGB sämtliche Vollmachten auf der Homepage des DFB abruf- 
bar. ${ }^{49}$ Der Hinweis hierauf soll ausreichen, um die Rechtsfolge des $\$ 174$ BGB vermeiden zu können. ${ }^{50}$

Der sich so ergebende Zweckzusammenschluss der beteiligten Vereine, Verbände und Gesellschaften ist im rechtlichen Sinne eine BGB-Gesellschaft i.S.d. $\mathbb{\$} \$ 705 \mathrm{ff}$. BGB. ${ }^{51}$ Dies hat Konsequenzen insbesondere für den gerichtlichen Rechtsschutz gegen ein Stadionverbot.

Das Stadionverbot soll nach dem Selbstverständnis der SVRL eine präventive Maßnahme sein. Es wird von den betroffenen Fans allerdings nicht ganz unnachvollziehbar als Sanktion empfunden, ${ }^{52}$ weil es abgesehen von einer repressiv konnotierten Sprachwahl und Regelungssystematik (s.u. unter D. IV. 4.) natürlich immer als Reaktion auf ein sicherheitsbeeinträchtigendes Verhalten ausgesprochen wird. Einen punitiven Charakter wird man dem Institut daher nicht gänzlich absprechen können: Den Fan künftig zu einwandfreiem Verhalten zu erziehen oder andere Fans damit von Fehlverhalten abzuschrecken, folgt spezial- und generalpräventiven Zwecken, die dem Wesen von Strafe entsprechen.

\section{Inhalt und Wirkung der SVRL}

In elf teils umfangreich ausgestalteten Paragraphen legen die SVRL auf sage und schreibe 14 DIN-A-4-Seiten die einzelnen Bedingungen rund um die Aussprache ${ }^{53}$ von Stadionverboten fest. Von besonderem Interesse sind dabei die Vorschriften über Entstehungs- und Beendigungsvoraussetzungen eines Stadionverbots, namentlich $\mathbb{S} \$ 4,5,6$ und 7 SVRL.

\section{Kategorien und Festsetzungsmöglichkeiten eines Stadionverbots}

Die verschiedenen Arten von Stadionverboten und deren jeweilige tatbestandlichen Voraussetzungen erschließen sich erst nach intensiverem Studium der SVRL. Die systematisch nicht immer geradlinige Konstruktion und auch die verschachtelten Tatbestandsmerkmale erschweren einen raschen und verständlichen Überblick über dieses aus Fansicht sensible Thema. Daher sollen hier anstelle eines bloßen Verweises auf die Normen die einzelnen Festsetzungsarten in einem systematischen Gesamtkontext eingebettet wiedergegeben werden.

Es ergeben sich unterschiedliche Einteilungsmöglichkeiten, obwohl in $\mathbb{} 5$ Abs. 2 SVRL eine einheitliche Unterteilung in die so bezeichneten Kategorien „A “, „B“ und „C“ versucht wird. Dabei steht „Kategorie A“ für einen „minderschweren Fall“ mit

49 http://www.dfb.de/index.php?id=503930.

50 LG Paderborn, Urt. v. 20.6.2008, Az. 2 O 10/08, SpuRt 2008, 124.

51 LG Duisburg, Urt. v. 22.7.2005, Az. 7 S 63/05 (juris); Orth, a.a.O. (s.o. Fn. 6), S. 139.

52 S.o. Fn. 10.

53 Einen Fachterminus zur Aussprache von Stadionverboten verwenden die SVRL nicht; häufig wird auch von „Festsetzung“ oder schlicht „Stadionverbot“" gesprochen. 
Verweis auf $\mathbb{} 4$ Abs. 2 SVRL, „Kategorie B“ für einen „schweren Fall“ mit Verweis auf $\mathbb{S} 4$ Abs. 3, 4 und 5 SVRL sowie „Kategorie C“ für einen „besonders schweren Fall“, ebenfalls mit Verweis auf $\mathbb{S} 4$ Abs. 3, 4 und 5 SVRL. Obwohl das unterschiedliche Attributieren der „Fälle“ erst auf Rechtsfolgenseite der SVRL erfolgt, bezieht es sich doch auf den Festsetzungsgrund und gerade nicht die Dauer des Stadionverbots als Rechtsfolge. ${ }^{54}$ Ungeachtet dieser Formalia lassen sich inhaltlich folgende Differenzierungen vornehmen:

\section{a) Festsetzung mit und ohne Ermessen}

Ein Unterteilungsmerkmal könnte zunächst der jeweils zugestandene Ermessensspielraum des Festsetzungsberechtigten sein. Dem Wortlaut nach zwingend „ist“ ein Stadionverbot nämlich gem. $\mathbb{S}$ Abs. 1 SVRL nur dann „zu verhängen“, wenn der Adressat bereits nachweislich „in einer die Menschenwürde verletzenden Art und Weise oder sicherheitsbeeinträchtigend aufgetreten ist. " Dagegen wird dem Wortlaut nach in $\mathbb{S} 4$ Abs. 2, 3, 4 und 5 SVRL dem Festsetzungsberechtigten ein Ermessen eingeräumt, welches allerdings nur bei Auslandsbezug gem. Abs. 5 frei („kann“) und im Übrigen intendiert („,soll“) ist. In der Praxis kann daher auch allenfalls in den Fällen von $\mathbb{} 4$ Abs. 5 SVRL von einem tatsächlichen Ermessensspielraum ausgegangen werden.

Möglich ist allerdings auch eine andere Lesart von $\mathbb{\int} 4$ SVRL, wonach Abs. 1 als generalklauselartige Einleitung der sodann aufgeführten Tatbestände dient. Darauf könnte der Wortlaut „in einem oder mehreren der im Folgenden aufgeführten Fälle“ hindeuten. Ist jedoch bereits einer der „aufgeführten Fälle“ - wie es Abs. 1 für seine eigene Anwendbarkeit voraussetzt - einschlägig, bedarf es keines Rückgriffs auf Abs. 1 mehr. Folglich wäre diese Klausel obsolet, es sei denn, man verbindet sie mit den ihr folgenden Tatbeständen. Dann wäre aber wegen der verpflichtenden Formulierung von Abs. 1 („,ist zu verhängen“) jede Stadionverbotsfestsetzung zwingend, mithin wären die ermessensbezogenen Formulierungen der Abs. 2 bis 5 gegen den ausdrücklichen Wortlaut der SVRL zu ignorieren.

Angesichts dieser Wertungswidersprüche ist Abs. 1 richtigerweise an seinen Bezügen zur Menschenwürde und zu nachgewiesenen Sicherheitsbeeinträchtigungen zu messen. Existiert eine dieser Tatbestandsvoraussetzungen zusätzlich zu den Voraussetzungen von Abs. 2 bis 5, wandelt sich das Ermessen des Festsetzungsberechtigten zu einer Festsetzungspflicht; i.Ü. bleibt es beim intendierten Ermessen. Im Ergebnis wird damit ein Stadionverbot grundsätzlich nach intendiertem Ermessen festgesetzt, wenn dieses nicht ausnahmsweise auf null reduziert ( $\mathbb{} 4$ Abs. 1 SVRL) oder aber für frei erklärt ( $\int 4$ Abs. 5 SVRL) wird.

54 Dies wird freilich erst und nur in $\ 5$ Abs. 2 UAbs. 2, der sich für den „,besonders schweren Fall“ auf bestimmte Umstände des Festsetzungsgrundes bezieht, erkennbar; mehr dazu sogleich unter B. III. 2. 


\section{b) Örtliches und überörtliches Stadionverbot}

Der Regelfall des Stadionverbots wird durch $\$ 1$ Abs. 4 SVRL als örtliches Stadionverbot definiert, denn das „Stadionverbot erstreckt sich grundsätzlich nur auf den befriedeten Bereich der Platz- oder Hallenanlage, in der das Hausrecht des das Stadionverbot Festsetzenden ausgeübt wird“. Gem. $\mathbb{} 4$ Abs. 2 SVRL soll es „bei Verstößen gegen die Stadionordnung ausgesprochen werden (minderschwerer Fall), soweit diese nicht mit Verstößen nach Absatz 3 in Verbindung stehen oder der Betroffene bisher nicht wiederholt sicherheitsbeeinträchtigend aufgefallen ist“". Der Wortlaut verdeutlicht bereits, dass es sich bei dieser Bestimmung um nichts Anderes als die originäre Ausübung des Hausrechts handelt, welche auf Grundlage eines bereits festgestellten und erwiesenen (sic!) Fehlverhaltens - den Verstoß gegen die Stadionordnung und damit die Nebenpflichten aus dem Stadionbesuchsvertrag ${ }^{55}$ - erfolgt. Es ist zugleich ein verstoßbegründetes Stadionverbot (dazu sogleich). Damit überrascht nicht, dass die Variante des örtlichen Stadionverbots nicht in der öffentlichen Kritik steht; sie soll auch hier keine weitere Beachtung finden.

Die Aussprache eines sog. „überörtlichen“ oder „bundesweiten“ Stadionverbots i.S.v. $\mathbb{S} 1$ Abs. 5 SVRL wird tatbestandlich zum einen von einem ,eingeleiteten Ermittlungs- oder sonstigen Verfahren“ ( $\$ 4$ Abs. 3 SVRL) auf Grund des Verdachts von einschlägigen Katalogverstößen ( $\$ 4$ Abs. 3 Nrn. 1 bis 15 SVRL) abhängig gemacht. Sie erfolgt also verdachtsbegründet (dazu ebenfalls sogleich). Zum anderen ist eine Festsetzung auch ohne Verfahrenseinleitung möglich bei bereits festgestellten, gleichermaßen katalogisiert aufgeführten Verstößen $(\mathbb{} 4$ Abs. 4 Nrn. 16 bis 19 SVRL) wie z.B. „schwerwiegenden Verstößen gegen die Stadionordnung“ nach Nr. 19. Damit entsprechen die Voraussetzungen der nicht an eine Verfahrenseinleitung geknüpften Variante des überörtlichen Stadionverbots strukturell jenen des örtlichen Stadionverbots; denn in beiden Fällen soll der Festsetzungsberechtigte erst tätig werden, wenn ein Fehlverhalten des Störers bereits nachgewiesen wurde. Das überörtliche Stadionverbot nach $\mathbb{} 4$ Abs. 4 SVRL ist folglich verstoßbegründet, wirft damit ebenso wenig Probleme auf und soll hier daher auch nicht weiter vertieft werden.

\section{c) Verdachtsbegründetes und verstoßbegründetes Stadionverbot}

Aus dem bisher Ermittelten entspringt eine weitere, so nicht in den SVRL aufgeführte, dafür jedoch in ihrer Tragweite nicht zu unterschätzende Differenzierungsmöglichkeit: die Unterscheidung von verdachts- und verstoßbegründeten Stadionverboten. Auf die verstoßbegründeten Stadionverbote fällt im Rahmen der SVRL formell wesentlich mehr Gewicht, während ein verdachtsbegründetes Stadi- 
onverbot nur als Spezialfall des überörtlichen Stadionverbots und nach den Voraussetzungen von $\$ 4$ Abs. 3 SVRL festgesetzt werden soll.

In der Rechtsanwendungswirklichkeit jedoch bildet es den Kernbereich der Festsetzungspraxis. Darüber hinaus ist es die einzige Variante des gesamten Themenkomplexes „Stadionverbot“, welche in der öffentlichen Wahrnehmung überhaupt unter der Bezeichnung „Stadionverbot“ diskutiert wird. Genau genommen müsste es aber als „bundesweites verdachtsbegründetes Stadionverbot“ bezeichnet werden. Eben diese Verknüpfung mit einem bloßen Verdacht, der sich typischerweise anhand der Einleitung eines staatsanwaltschaftlichen Ermittlungsverfahrens kennzeichnet, bietet einen großen Nährboden für den rechtswissenschaftlichen Diskurs. Verstärkt wird dies angesichts des konstruktiven und inhaltlichen unmittelbaren strafrechtlichen und strafverfahrensrechtlichen Bezuges von $\mathbb{} \$$ Abs. 3 SVRL, der die Verdachtsgrundlage des eingeleiteten Ermittlungsverfahrens in seinem Katalog auf ausgewählte Regelbeispiele ${ }^{56}$ von (jedenfalls ganz überwiegend) ${ }^{57}$ strafrechtlichen Vorschriften stützt.

\section{Dauer und Aufhebung eines Stadionverbots}

Wurde ein Stadionverbot erst einmal ausgesprochen, richtet sich seine Dauer grundsätzlich nach den durch die SVRL vorgegebenen zeitlichen Grenzen, wenn es nicht ausnahmsweise zu seiner Reduzierung, Aussetzung oder Aufhebung kommt.

\section{a) Grundsätzliche zeitliche Grenzen}

Die Mindestdauer eines Stadionverbots beträgt gem. $\$ 5$ Abs. 1 SVRL eine Woche. Die Höchstdauer richtet sich dagegen gem. $\$ 5$ Abs. 2 SVRL nach der Einstufung des mittels der Kategorien „A“, „B“ und „C“ nach Schwere gestaffelten Grundes, auf welchem das Stadionverbot basiert. ${ }^{58}$

Minderschwere Fälle werden ausschließlich bei einem örtlichen Stadionverbot ( $\$ 4$ Abs. 2 SVRL) angenommen, sind also in der praktischen Anwendung kaum relevant, auch wenn sie sich erfreulich eindeutig ermitteln lassen. Schwieriger fällt dagegen die Differenzierung zwischen schweren und besonders schweren Fällen, weil $\$ 5$ Abs. 2 SVRL bei beiden Kategorien gleichermaßen auf $\$ 4$ Abs. 3, 4 und 5 SVRL verweist. Folglich fußt jedes bundesweite Stadionverbot, sei es verdachts- oder verstoßbegründet, entweder auf einem schweren oder besonders schweren Fall. Den einzigen Hin-

56 Dass es sich um Regelbeispiele handelt, ist zum einen aus der Formulierung „insbesondere in folgenden Fällen“ sowie der den Katalog abschließenden Nr. 15 - „Sonstige schwere Straftaten im Zusammenhang mit Fußballveranstaltungen " - zu schließen.

57 Lediglich Nr. 14 („Einbringen und / oder Abbrennen von pyrotechnischen Gegenständen“) kann nach landesrechtlichen Vorschriften auch als bloße Ordnungswidrigkeit ausfallen (z.B. $\$ 17$ Abs. 1 lit.g LImSchG NRW), wenn nicht eine Gefährdung von Leben, Gesundheit oder Eigentum hinzutritt (z.B. \18 Abs. 1 LImSchG NRW),

58 S. bereits oben unter B. III. 1. 
weis auf Unterscheidungskriterien zwischen den Kategorien „B“ und „C“ liefert

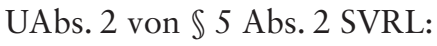

„Ein besonders schwerer Fall liegt insbesondere vor, wenn der Betroffene wegen besonderer Intensität in einem der in $\$ 4$ Abs. 3, 4 und 5 aufgeführten Fälle aufgefallen ist und/oder keinerlei Einsicht zeigt." 59

Dagegen orientiert sich $\mathbb{} 5$ Abs. 1 SVRL hinsichtlich der individuellen Dauer (nicht aber der Höchstdauer!) eines Stadionverbots unabhängig von seiner Kategorie an gängigen Strafzumessungsregeln der strafrechtsjustiziellen Praxis, indem Schwere des Einzelfalls, Folgen einzelner Taten, das Alter des Betroffenen, seine Einsichtsfähigkeit und Stellungnahmen Dritter Beachtung finden, mithin Tatunwert- und Schuldgedanken ausschlaggebend sein sollen. ${ }^{60}$

Bei Fällen von „Kategorie A“ läuft das Stadionverbot höchstens „bis zum 30. Juni des ersten Jahres, das auf die laufende Spielzeit folgt“. Diese recht umständliche Formulierung ist zum Teil der Tatsache geschuldet, dass sich der Kalender des Fußballs nach der jeweiligen Saison richtet, welche jedenfalls in den großen europäischen Fußballverbänden am 1. Juli eines Jahres beginnt und zum 30. Juni des Folgejahres endet. Der maximale Ablauf eines Stadionverbots nach „Kategorie A“ gilt damit immer bis zum Ende der aktuellen Saison plus der gesamten darauf folgende Saison. Dies hat zur Folge, dass je nach Festsetzungszeitpunkt ${ }^{61}$ des Stadionverbots die Höchstdauer zwischen genau einem Jahr (bei Aussprache am 30. Juni, mithin des letzten Tages der laufenden Spielzeit) und zwei Jahren minus einem Tag (Aussprache am 1. Juli, mithin des ersten Tages der laufenden Spielzeit) variieren kann.

Nach gleichem Muster errechnet sich die jeweilige Höchstdauer für Stadionverbote nach „Kategorie B“ („,bis 30. Juni des zweiten Jahres, das auf die laufende Spielzeit folgt“) und „Kategorie C“ („,bis 30. Juni des dritten Jahres, das auf die laufende Spielzeit folgt"); ebenso ungleich kann also je nach Festsetzungszeitpunkt die jeweilige Höchstdauer ausfallen.

\section{b) Reduzierung, Aussetzung, Aufhebung}

Neben den grundsätzlichen zeitlichen Vorgaben eröffnen die SVRL in $\mathbb{} 6$ und $\mathbb{} 7$ auch die Möglichkeit, bereits ausgesprochene Stadionverbote in ihrer Dauer zu reduzieren, sie auszusetzen oder gänzlich aufzuheben. Dabei sind Reduzierung und Aufhebung mit und ohne Ermessen in $\$ 6$ SVRL nach klaren Vorgaben geregelt. Demgegenüber sind Reduzierung, Aufhebung und zusätzlich - einem „Stadionverbot

59 Hervorh. durch die Verf.

60 Instruktiv statt vieler Schäfer/Sander/Gemmeren, Praxis der Strafzumessung, 4. Aufl. München 2008, insb. Rn. $310 \mathrm{ff}$.

61 Aus dem Rechtsgedanken von $\mathbb{\$} 8$ Abs. 2 SVRL, wonach im Zweifel eine förmliche schriftliche Zustellung zu erfolgen hat, dürfte der für den Beginn des Stadionverbots relevante Festsetzungszeitpunkt im Tag der Zustellung liegen. Eine ausdrückliche Regelung hierzu existiert jedoch nicht. 
auf Bewährung“ gleich - Aussetzung auf Grundlage einer Ermessensentscheidung nach den umfangreichen strafrechtlich orientierten ${ }^{62}$ und zugleich oft unbestimmten Vorgaben des $\mathbb{} 7$ SVRL möglich.

Hinsichtlich der Aufhebung eines verdachtsbegründeten bundesweiten Stadionverbots liefert $\$ 6$ SVRL inhaltlich eindeutige Antworten: Wird das Ermittlungsverfahren später nach $\mathbb{S} 170$ Abs. 2 StPO eingestellt, so ist das Stadionverbot gem. $\mathbb{S} 6$ Abs. 1 erster Spiegelstrich SVRL wieder aufzuheben. Dagegen soll es noch einmal auf Bestand und Dauer überprüft werden, wenn das Verfahren nach $\mathbb{\$} 153$ StPO eingestellt wurde ( $\mathbb{6}$ Abs. 2 erster Spiegelstrich SVRL). Eine Überprüfung lediglich der Dauer kommt hingegen in Betracht, wenn das Verfahren nach $\mathbb{} 153$ a StPO eingestellt wurde ( $\int 6$ Abs. 2 zweiter Spiegelstrich SVRL). ${ }^{63}$

Für einen Betroffenen, der mit einem Stadionverbot wegen eines laufenden Ermittlungsverfahrens beispielsweise wegen Landfriedensbruchs ( $\$ 4$ Abs. 3 Nr. 7 SVRL) belegt wurde, ist es bei späterer Verfahrenseinstellung damit von entscheidender Bedeutung, nach welcher strafprozessualen Norm die Staatsanwaltschaft vorgeht.

\section{3. Übrige Bestimmungen der SVRL}

Die übrigen Bestimmungen der SVRL beschränken sich auf Regelungen zu Verfahren und Form der Festsetzung bzw. Verwaltung eines Stadionverbots. Zu nennen sind in diesem Zusammenhang einerseits die Möglichkeit der Anhörung des Betroffenen nach $\mathbb{S} 5$ a SVRL, welche sich jedoch auch bei Ausbleiben nicht auf den Verfahrensinhalt auswirkt. Andererseits wird für die wesentlichen Verfahrensschritte die Schriftform angeordnet, was insbesondere für die Bekanntgabe durch nachweisbare Zustellung gilt (vgl. $\int 8$ SVRL).

Erwähnenswert ist zudem, dass nach $\mathbb{1} 10$ SVRL die Verwaltung und effektive Durchsetzung eines festgesetzten Stadionverbots mittels einer dafür eingerichteten Zentralstelle erleichtert wird. Sämtliche relevanten Daten werden regelmäßig zwischen der Zentralstelle, den Vereinen, dem DFB, der DFL und auch der zuständigen Polizei sowie der Bundespolizei ausgetauscht. Dank dieser Vernetzung kann ein Stadionverbot besonders wirksam realisiert werden, insbesondere auch im Hinblick auf die genannte Durchbrechung des Kontrahierungszwangs beim Eintrittskartenverkauf der Bundesligavereine. In der Praxis teilt also regelmäßig die Polizeibehörde den Vereinen mit, dass ein Ermittlungsverfahren gegen eine Person z.B. wegen Landfriedensbruchs eingeleitet wurde, fügt eine kurze Sachverhaltsdarstellung bei und emp-

$62 \sqrt{ } 7$ Abs. 1 SVRL spricht auch von einer „Tat“, während Abs. 2 typische Bewährungsgedanken wiedergibt: „Die Auflagen (z.B. über Aufenthaltsort, Meldepflichten, Mitwirkung an sozialen Aufgaben) sollen gewährleisten, dass der Betroffene wieder integriert wird und keine sicherheitsbeeinträchtigenden Taten während einer Fußballveranstaltung begehen kann.“

63 Diese Möglichkeit wird kaum diskutiert und führt bei einer Debatte um die Rechtslage zum Stadionverbot als solchem nicht weiter, sie soll daher nicht weiter beachtet werden. Gleichwohl ist (auch) diese Bestimmung der SVRL revisionswürdig, s. unten unter E. 
fiehlt oder drängt den nach den SVRL zuständigen Verein, gegen den Betroffenen ein Stadionverbot festzusetzen. ${ }^{64}$ Diese Datenübermittlung dürfte nach $\$ 16$ Abs. 1 Nr. 1 BDSG zulässig sein, weil die Weitergabe dieser Informationen - und die daraus resultierende Verhängung eines Stadionverbots - aus polizei-präventiven Erwägungen sinnvoll ist und damit auch zur Erfüllung der in der Zuständigkeit der übermittelnden Stelle liegenden Aufgaben (nämlich der Polizeibehörden) erforderlich sein dürfte.

\section{Kritik}

Die Regelungsmechanismen der SVRL bieten nicht wenig Anlass zur Kritik, welche sich nicht nur auf einen unter rechtlichen Aspekten zweifelhaften Inhalt beschränkt, der aber gleichwohl hierin den Kern der auch (sport-)gesellschaftlich emotional aufgeladenen Debatte ausmacht.

\section{Zweifelhafte Rechtsverbindlichkeit}

Nach klassischen vereinsrechtlichen Ansätzen wäre die Rechtsverbindlichkeit der SVRL für ihre Adressaten problematisch. Nur der Ligaverband ist Mitglied des DFB, der sie als Ordnung erlässt. Die Vereine der Bundesliga und 2. Bundesliga sind Mitglieder des Ligaverbands. Die Vereine der 3. Liga und der Regionalligen sind Mitglieder ihrer Landesverbände. Sie wären damit unmittelbar der Wirkung der SVRL nicht unterworfen. Angesichts neuerer Diskussionen darf auch ihre Bindung an das gesamte DFB-Recht über die bekannten dynamischen Verweisungen in ihren Satzungen oder Unterwerfungserklärungen jedenfalls zweifelhaft erscheinen. ${ }^{65} \mathrm{Da}$ rüber hinaus erscheint die gesamte Materie alleine unter dem Gesichtspunkt, dass ein auf ihrer Grundlage erteiltes Stadionverbot für den Betroffenen letztlich einen Grundrechtseingriff darstellen kann (s.u. unter D. II. 2.), so wesentlich zu sein, dass diese Regelung nicht allein in einer unter der Satzung stehenden Ordnung, sondern wenigstens ihrem wesentlichen Gepräge nach in der Verbandssatzung geregelt sein muss. Auch wäre es wegen der enormen Bedeutung nur sachgerecht, dieser Regelung durch das entsprechende Votum eines DFB-Bundestages hilfreiche und notwendige Legitimation zukommen zu lassen. Bedenken bereitet zudem, dass so wesentliche und wichtige Fragen nicht von einem anerkannten DFB-Gremium, etwa dem Präsidium, beschlossen werden, sondern die Beschlussfassung hierüber an ein intransparent und nicht einmal durch den DFB-Bundestag besetztes Gremium, nämlich eine bloße „Kommission“, delegiert wird.

Da aber die Mustervorlage nach $\$ 1$ Abs. 5 SVRL die Formulierung enthält, dass [der Verein] ,ausdrücklich $\mathbb{3 1}$ der Richtlinien zur Verbesserung der Sicherheit bei Bundesspielen und die auf dieser Grundlage vom DFB erlassenen weiteren Richtlinien

65 Vgl. zuletzt Heermann, ZHR 174 (2010), 250 ff.; Orth/Pommerening, SpuRt 2010, 222. 
[...] mit den darin enthaltenen Regelungen über das bundesweit wirksame Stadionverbot sowohl in formeller als auch in materieller Hinsicht als rechtswirksam und für sich verbindlich “ anerkennt, gelten die Stadionverbotsrichtlinien im Wege des im Rahmen der Privatautonomie zweifellos zulässigen Regelanerkennungsvertrags für die Festsetzungsberechtigten, weil gerade jene Anerkennungserklärung von allen Festsetzungsberechtigten zu Beginn einer jeden Spielzeit formularmäßig abgegeben wird. Die hier am Rechtssetzungsverfahren die SVRL betreffend geäußerte Kritik bleibt damit juristisch letztlich ohne Folge.

\section{Die Einleitung eines Ermittlungsverfahrens als Grundlage}

Die bloße Einleitung eines staatsanwaltlichen Ermittlungsverfahrens kann und darf nicht zur Grundlage für die Erteilung eines Stadionverbots gemacht werden, wie dies in $\mathbb{} 4$ Abs. 3 SVRL für das verdachtsbegründete Stadionverbot angeordnet wird. ${ }^{66}$ Hierbei wird nicht verkannt, dass das Stadionverbot als präventive Maßnahme lediglich eine Gefahrenprognose zu Lasten des Betroffenen begründet; eine solche beruht regelmäßig auf einem nach Ort und Datum nachgewiesenen Fehlverhalten („dokumentierte Legende“). ${ }^{67}$ Auf die Einleitung eines Ermittlungsverfahrens kann dabei aber allein nicht abgestellt werden. Denn hierfür ist lediglich ein auf Tatsachen beruhender Verdacht, der sog. Anfangsverdacht, erforderlich. ${ }^{68}$ Hierbei ist allerdings entscheidend, dass der Anfangsverdacht die schwächste Form strafrechtlichen Verdachts ist; ${ }^{69} \mathbb{} \$ 152$ Abs. 2 StPO lässt nämlich bloß „zureichende tatsächliche Anhaltspunkte" ausreichen. Für einen Anfangsverdacht genügt, dass auch nur entfernte Indizien - nicht aber bloße Vermutungen - bestehen, die das Vorliegen einer Straftat objektiv für möglich erscheinen lassen. ${ }^{70}$ Dies stellte bereits Breucker in seinem vom BGH zu Grunde gelegten Aufsatz fest: „Allein der abstrakte Umstand als solcher, dass gegen den Betroffenen einmal ein staatliches Verfahren eingeleitet wurde, vermag eine Gefahrenprognose nicht zu begründen. "71 Soweit also das verdachtsbegründete Stadionverbot lediglich auf einem Anfangsverdacht beruht, ist es nach rechtstaatlichem Verständnis kaum haltbar.

\section{Die Art der Verfahrenseinstellung als Vorgabe für die Aufhebung eines Stadionverbots} Diese Fehlanknüpfung von $\mathbb{} 4$ Abs. 3 SVRL frisst sich in $\$ 6$ SVRL fort, indem die von der Staatsanwaltschaft verfügte Art der Verfahrenseinstellung $-\mathbb{\$} 170$ Abs. 2

66 „Bedenklich“ findet dies auch Heermann, a.a.O. (s.o. Fn. 4).

67 Breucker, a.a.O. (s.o. Fn. 6), 135.

68 Dies erkennt auch der BGH, a.a.O. (s.o. Fn. 4), Rn. 21, an.

69 Anders z.B. der „einfache“ Tatverdacht für die Durchsuchung (\$102 StPO), „genügenden Anlass“ (i.S. einer gewissen Verurteilungswahrscheinlichkeit) für die Anklageerhebung ( $\$ 170 \mathrm{Abs.} 1 \mathrm{StPO}$ ), der hinreichende Tatverdacht für die Eröffnung des Hauptverfahrens ( $\$ 203$ StPO) oder gar der dringende Tatverdacht $(\mathbb{S} 112$ Abs. 1 Satz 1 StPO) für die Anordnung der Untersuchungshaft.

70 Meyer-Goßner, StPO, 53. Aufl., 2010, $\$ 152$, Rn. 4; KarlsruherKomm-Schoreit, 6. Aufl., 2008, $\$ 152$, Rn. 28.

71 Breucker, a.a.O. (s.o. Fn. 6), 135. 
oder $\$ 153 \mathrm{StPO}$ - als maßgeblich für die Entscheidung über Aufrechterhaltung oder Aufhebung eines Stadionverbots erhoben wird. ${ }^{72}$

\section{a) Staatsanwaltliche Einstellungspraxis}

Diese in $\$ 6$ SVRL geregelte Diskrepanz zu Lasten des Beschuldigten spiegelt keinesfalls die Realität der staatsanwaltlichen Praxis im Umgang mit Verfahrenseinstellungen nach $\mathbb{S} 170$ Abs. 2 und $\mathbb{S} 153$ StPO wider. ${ }^{73}$ Da der Beschuldigte in beiden Einstellungsfällen nicht beschwert ist, ${ }^{74}$ kann man der häufig unter hohem Arbeitspensum leidenden Staatsanwaltschaft einen nicht immer exakt dem Gesetz folgenden, trennscharfen Umgang mit diesen beiden Prozessvorschriften auch sicher nicht vorwerfen. ${ }^{75}$ Zudem erfolgt eine Einstellung nach $\$ 153$ StPO bekanntlich unabhängig von einer tatsächlich festgestellten Schuld. Denn diese Einstellungsart unterstellt eine mögliche Schuld - und zwar nur unter der weiteren Unterstellung, der Tatvorwurf sei zutreffend - ohne konkreten und objektiven Nachweis als gering auf Grundlage einer durch die Staatsanwaltschaft getätigten Wahrscheinlichkeitsbeurteilung. ${ }^{76}$ Selbst wenn sich eine für den Beschuldigten günstigere Einstellungsart, z.B. nach $\mathbb{1 7 0}$ Abs. 2 StPO, ergeben könnte, ist diese zusätzliche Klärung nicht geboten; ${ }^{77}$ auch und schon deshalb erweist sich die punktgenaue Differenzierung zwischen beiden Einstellungsarten in $\$ 6$ SVRL als praxisignorant und unhaltbar.

\section{b) Fehlende Rechtsmittel}

Etwas Anderes könnte sich allenfalls dann ergeben, wenn der Betroffene gegen die Einstellungsentscheidung der Staatsanwaltschaft Rechtsmittel einlegen könnte. ${ }^{78} \mathrm{Er}$ hätte dann die Möglichkeit, einen anderen Einstellungsgrund zu erstreiten und damit die Bezugstatsache für die SVRL zu seinen Gunsten zu verändern. Eine verwaltungsgerichtliche Klage scheidet jedoch mangels öffentlich-rechtlicher Streitigkeit aus. ${ }^{79}$ Im Rahmen einer ebenfalls denkbaren gerichtlichen Überprüfung nach $\$ 23$ EGGVG ist bereits die Qualität der staatsanwaltlichen Einstellungsverfügung als Justizverwaltungsakt umstritten, jedenfalls sind aber Maßnahmen der Staatsanwaltschaft hierbei grundsätzlich unanfechtbar. ${ }^{80}$ Schließlich wird eine - bereits als solche um-

72 S. bereits oben unter B. III. 2. b).

73 Diesen wichtigen Aspekt verkennt Breucker, SpuRt 2010, 31 (Punkt 2).

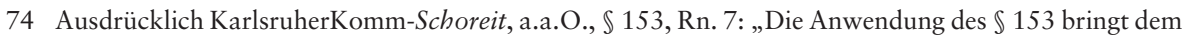
Beschuldigten keine Nachteile“; vgl. auch Meyer-Goßner, a.a.O., $\$ 153$, Rn. 27.

75 S. dazu auch die verfassungsrechtlichen Erwägungen unter D. II. 3. b).

76 Meyer-Goßner, a.a.O., $\$ 153$, Rn. 3; Schäfer/Sander/Gemmeren, a.a.O. (s.o. Fn. 60), Rn. 16. Die Verbindung zwischen Unschuldsvermutung und $\mathbb{} 153$ StPO ist i.Ü. spätestens seit BVerfGE 82, 106, grundrechtlich garantiert.

77 Meyer-Goßner, a. a. O; KarlsruherKomm-Schoreit, a.a.O.

78 Umfassend dazu Heinrich, NStZ 1996, 110.

79 Heinrich, a.a.O. 110, 115 m.w.N.

80 Vgl. Meyer-Goßner, a.a.O., $\$ 23$ EGGVG, Rn. 9; umfassend KarlsruherKomm-Schoreit, a.a.O., $\mathbb{} 23$ EGGVG, Rn. $31 \mathrm{ff}$. 
strittene - analoge Anwendung von $\$ 23$ EGGVG allenfalls bei objektiv willkürlichen Verfahrenseinstellungen diskutiert, ${ }^{81}$ hat aber mangels Gerichtspraxis keine tatsächlichen Erfolgsaussichten. Eine Dienstaufsichtsbeschwerde oder eine Gegenvorstellung gegen die staatsanwaltliche Entscheidung ${ }^{82}$ schließlich haben als bloße Petitionen keinen Rechtsmittelcharakter, müssen keine begründete Überprüfung zur Folge haben $^{83}$ und werden deswegen regelmäßig nicht zu einer Abänderung führen.

\section{c) Zwischenergebnis}

Aus all diesen Gründen können auch aus der Art der Verfahrenseinstellung nach $\$ 170$ Abs. 2 oder $\$ 153$ StPO allein keine Rückschlüsse auf eine Gefahrenprognose gezogen werden, auf welche es bei der Festsetzung eines Stadionverbots nach wie vor und ausschließlich ankommt.

\section{Weitere Defizite der SVRL}

Neben diesen Mängeln speziell beim verdachtsbegründeten Stadionverbot wissen die SVRL auch unter anderen Gesichtspunkten mitunter wenig zu überzeugen.

So wiederholen die SVRL gebetsmühlenartig einen rein hausrechtlich-präventiven Charakter eines Stadionverbots. Diese Grundvoraussetzung, welche das Konstrukt der SVRL dogmatisch zusammenhält, wird jedoch durch die sich in den SVRL widerspiegelnde Normsetzungstechnik konterkariert. Die Konstruktion der einzelnen Bestimmungen folgt nämlich vorwiegend den üblichen strafrechtlichen Mustern, wie z.B. die Verwendung von Regelbeispielen ${ }^{84}$ oder die Abhängigkeit der "Schwere“ eines „Falls“ für die Dauer des Stadionverbots ( $\$ 5$ Abs. 2 SVRL) belegen. Diese Kategorisierung richtet sich bei Lichte betrachtet nur nach der Schwere des Festsetzungsgrundes und muss sich aus zwingend logischen Gründen für ein verlässliches Abwägen der Schwere auf Rechtsfolgenseite auf ein nachgewiesenes Vorverhalten des Betroffenen beziehen, was bei einem verdachtsbegründeten Stadionverbot schlicht unmöglich ist. Pointiert wird dieser Widerspruch in $\$ 7$ SVRL, welcher schließlich auch inhaltlich von einem punitiven Charakter - hier geht es ausdrücklich um eine „Tat“! - ausgeht und dabei auf typische Strafzumessungsregeln und Bewährungsmaßnahmen zurückgreift. ${ }^{85}$

In dieses Bild passt auch das Abstellen auf eine fehlende Einsichtigkeit des Betroffenen in $\$ 5$ Abs. 2 SVRL; die Bestimmung unterstellt einen gewissen Grad an Schuld und soll gleichzeitig unterschiedslos auch bei einem verdachtsbegründeten Stadionverbot maßgeblich sein. Abgesehen davon, dass unter diesen Voraussetzungen nur

81 Heinrich, a.a.O. 110, $113 \mathrm{ff}$.

82 KarlsruherKomm-Schoreit, a.a.O., $\$ 153$, Rn. 36, m.w.N.

83 Meyer-Goßner, a.a.O., Vor $\$ 296$, Rn. 22 ff.; KarlsruherKomm-Paul, a.a.O., Vor $\$ 296$, Rn. 4.

84 S. oben unter B. III. 1. c).

85 S. oben unter B. III. 2. b). 
schwerlich von einer reinen Präventivmaßnahme gesprochen werden kann, ${ }^{86}$ beschleicht hier nicht nur den wenig wohlwollenden Betrachter das Gefühl, dass Betroffene eines Stadionverbots zu Gunsten einer Reduktion des - man müsste sagen: - „Strafmaßes“ zu einem Schuldgeständnis möglicherweise ohne zu Grunde liegender Tat gedrängt werden sollen.

\section{Zivilrechtliche Grundlagen}

Zivilrechtliche Grundlage des Stadionverbots ist das Hausrecht. Es ist ein Hausverbot, das sich auf ein Stadion bezieht. Das Hausrecht steht auch Fußballstadieninhabern zu. ${ }^{87}$ Nach zutreffender, heute wohl allgemeiner Meinung ist das Hausrecht keine einheitliche Befugnis, sondern ein Konglomerat verschiedener Rechte unterschiedlicher Reichweite, die auf dem Eigentum und/oder dem Besitz beruhen. ${ }^{88} \mathrm{Ge}-$ danklich ergibt es sich aus der Befugnis nach $\$ 903$ BGB, mit der im Eigentum stehenden Sache nach Belieben zu verfahren, insbesondere also auch jede Einwirkung auf eigene Sachen auszuschließen. ${ }^{89}$ Darüber hinaus gewährt auch der bloße (berichtigte oder auch unberechtigte) Besitz an einer Sache teils weitgehende Schutzrechte. Die zur Erteilung eines Hausverbots in Betracht kommenden Anspruchsgrundlagen sind damit die folgenden:

\section{Anspruch aus $\S 1004$ Abs. 1 Satz 2 (analog), 823 Abs. 1 BGB}

Ist der Veranstalter als Stadionbetreiber zugleich Stadioneigentümer, kann er Unterlassungsansprüche unmittelbar aus $\mathbb{} 1004$ Abs. 1 Satz 2 BGB geltend machen, um ein Stadionverbot durchzusetzen. Ist er als Mieter, Pächter oder durch sonstigen Nutzungsvertrag Berechtigter „nur“ bloßer Besitzer des Stadions, kann er sich aus S $\$ 1004$ Abs. 1 Satz 2 analog, 823 Abs. 1 BGB auf den gleichen Unterlassungsanspruch berufen.

\section{Beeinträchtigung von Eigentum oder einem sonstigen „absoluten Recht“ (petitorisch)}

Es muss zunächst eine Beeinträchtigung von Eigentum oder einem sonstigen „absoluten Recht“ vorliegen. Denn entsprechend $\$ 1004$ Abs. 1 BGB sind alle absoluten Rechte durch den Unterlassungsanspruch geschützt, die nach den $\mathbb{S} \mathbb{S} 823,824,825$,

86 Klesczewski, a.a.O. (s.o. Fn. 5), S. 254, sieht darin zu Recht einen „punitiven Zug“.

87 BGH, Urt. v. 8.11.2005, Az. KZR 37/03, NJW 2006, 378 (II.).

88 BGH, Urt. v. 20.1.2006, Az. V ZR 134/05 NJW 2006, 1054; Christensen, Taschenkontrolle im Supermarkt und Hausverbot - BGHZ 124, 39, JuS 1996, 873 m.w.N.; vgl. auch MünchKomm-Baldus, Bd. 6, 5. Aufl., 2009, $\$ 1004$, Rn. 6.

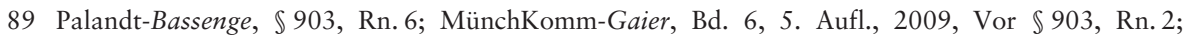
Staudinger-Seiler, BGB (Neubearbeitung 2002), \903, Rn. 11; zur grundsätzlichen Zulässigkeit des Hausverbots für Örtlichkeiten, die der Inhaber dem generellen Zutritt geöffnet hat - Kaufhaus -, vgl. BGH, Urt. v. 3.11.1993, Az. VIII ZR 106/93, BGHZ 124, 39. 
826 BGB mit deliktischem Schutz ausgestattet sind. ${ }^{90}$ Hierzu gehört der Besitz, auch im Verständnis des Besitzes zur Nutzung und zum Gebrauch. ${ }^{91}$

Eine Beeinträchtigung des Eigentums oder des Besitzes an einem Stadion, derer sich der Berechtigte erwehren kann, liegt schon bei der tatsächlichen Benutzung, insbesondere also beim Betreten durch Menschen vor. ${ }^{92}$ Denn unter Beeinträchtigung im Sinne dieser Vorschrift wird jeder dem Inhalt des Eigentums widersprechende Eingriff in die rechtliche oder tatsächliche Herrschaftsmacht des Eigentümers ( $\mathbb{9} 903$ BGB) verstanden; sie liegt selbst dann vor, wenn die Einwirkung auf die Sachsubstanz unschädlich ist. ${ }^{93}$ Damit stellt das Betreten eines Stadions durch einen Fan eine abwehrfähige Beeinträchtigung dar.

Während $\$ 1004$ Abs. 1 Satz 1 BGB auf die Beseitigung einer gegenwärtigen Beeinträchtigung gerichtet ist, kann zur Rechtfertigung eines Stadionverbots nur der in die Zukunft gerichtete Unterlassungsanspruch des $\mathbb{\$} 1004$ Abs. 1 Satz 2 BGB herangezogen werden. Er ist auch auf das richtige Anspruchsziel gerichtet: Geschuldet wird nicht nur bloße Untätigkeit, sondern auch ein Verhalten, das den Nichteintritt der drohenden Beeinträchtigung bewirkt. ${ }^{94}$ Im Falle des Stadionverbots darf also der Fan den Stadionbereich nicht mehr betreten.

\section{Geltendmachung im fremden Namen}

Die in den SVRL im Fall des bundesweiten Stadionverbots vorgesehene Geltendmachung eines Unterlassungsanspruchs aus $\$ 1004$ Abs. 1 Satz 2 BGB durch einen Anderen (vgl. oben) ist in rechtlicher Hinsicht nicht zu beanstanden. Zwar ist der Anspruch aus $\$ 1004$ Abs. 1 BGB nicht abtretbar. ${ }^{95}$ Ein Dritter kann jedoch zur Geltendmachung im eigenen Namen ermächtigt werden. ${ }^{96}$ Eine unwirksame Abtretung ist in der Regel in eine solche Ermächtigung umzudeuten. ${ }^{97}$ Außerdem wählen die SVRL konstruktiv den Weg der Stellvertretung, sodass der Festsetzungsberechtigte für die anderen Stadioninhaber den ihnen selbst zustehenden Anspruch aus $\$ 1004$ Abs. 1 Satz 2 BGB als Stellvertreter gemäß $\$ \mathbb{S} 164 \mathrm{ff}$. BGB ausspricht. Materielle Wirksamkeitsbedenken unter dem Blickwinkel des Stellvertretungsrechts ergeben sich insoweit, abgesehen von der Vorlageobliegenheit für die Vollmacht nach $\$ 174$ BGB (vgl. oben B. II. 2.), nicht.

90 MünchKomm-Baldus, a.a.O., $\$ 1004$, Rn. 26; Palandt-Bassenge, $\mathbb{1} 1004$, Rn. 4; Staudinger-Gursky, BGB (Neubearbeitung 2006), \$1004, Rn. 16.

91 Palandt-Bassenge, $\mathbb{8} 823$, Rn. 13; Staudinger-Gursky, a.a.O., $\mathbb{1} 1004, \mathrm{Rn} .15$

92 MünchKomm-Baldus, a.a.O., \1004, Rn. 43; Palandt-Bassenge, \$1004, Rn. 8; BayObLG, Urt. v. 25.5.2004, Az. 12 RR 2/03, BayObLGZ 2004, 138, Rn. 21 (juris).

93 BGHZ 111, 158.

94 Palandt-Bassenge, $\mathbb{S} 1004$, Rn. 33; Staudinger-Gursky, a.a.O., $\mathbb{S} 1004$, Rn. 210.

95 Palandt-Bassenge, $\mathbb{S} 1004$, Rn. 2; Staudinger-Busche, BGB (Neubearbeitung 2005), $\mathbb{S} 399$, Rn. 29.

96 Palandt-Bassenge, a.a.O.; Staudinger-Busche, a.a.O.

97 OLG Düsseldorf, Urt. v. 25.10.1995, Az. 11 U 8/94, ZMR 1996, 28. 


\section{Keine Duldungspflicht, $§ 1004$ Abs. 2 BGB}

Der Anspruch aus $\mathbb{} 1004$ Abs. 1 BGB ist ausgeschlossen, wenn der Eigentümer zur Duldung der Beeinträchtigung verpflichtet ist, $\mathbb{S} 1004$ Abs. 2 BGB. Eine solche Duldungspflicht kann sich insbesondere aus einer vertraglichen Vereinbarung ergeben. ${ }^{98}$ Diese könnte sich für den von einem Stadionverbot betroffenen Fan aus einer ihm verkauften Stadiondauerkarte ergeben, denn diese berechtigt ihn, alle kommenden Heimspiele des ausgebenden Vereins im jeweiligen Stadion zu besuchen. Im Falle eines berechtigten Stadionverbots kann aber eine solche Duldungspflicht nicht bestehen. Denn nach allgemeiner Auffassung stellen sicherheitsbeeinträchtigende Umstände, die zum Stadionverbot führen können, einen wichtigen Grund i.S.d. $\mathbb{S} 314$ Abs. 1 BGB dar, die den Verein zur außerordentlichen Kündigung des zu Grunde liegenden Dauerschuldverhältnisses berechtigt. ${ }^{99}$

\section{Wiederholungsgefahr}

Endlich muss aber für das Entstehen des Anspruchs nach $\ 1004$ Abs. 1 BGB „Wiederholungsgefahr" vorliegen.

\section{a) Herrschende Meinung}

Wiederholungsgefahr, als materielle Anspruchsvoraussetzung, ist die auf Tatsachen gegründete, objektiv ernstliche Besorgnis weiterer Störungen. ${ }^{100}$ Trotz des Gesetzeswortlauts („weitere“) lässt die Rechtsprechung unter dem Gesichtspunkt effektiven Rechtsschutzes auch eine erstmals zu befürchtende Beeinträchtigung ausreichen (sog. Erstbegehungsgefahr; „vorbeugender Unterlassungsanspruch“). ${ }^{101}$

\section{b) Meinungen zur Gefahrprognose für das Stadionverbot}

An diesen grundsätzlichen Anforderungen an die Wiederholungs- oder Erstbegehungsgefahr, also eine Prognoseentscheidung dahin, ob zukünftig von dem betroffenen Fan wieder eine Gefahr anlässlich von Stadionbesuchen ausgehen wird, ändert sich zunächst nichts. Dies sieht der BGH im Ausgangspunkt ebenso:

„Ein sachlicher Grund für ein Stadionverbot besteht daher, wenn aufgrund von objektiven Tatsachen, nicht aufgrund bloßer subjektiver Befürchtungen, die Gefahr besteht, dass künftige Störungen durch die betreffenden Personen zu besorgen sind. Eine derartige Gefahr wird regelmäßig bei vorangegangenen

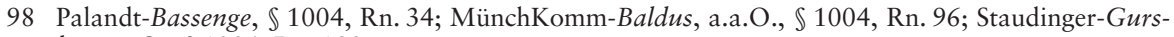
ky, a.a.O., $\mathbb{S} 1004, \mathrm{Rn} .193$.

99 BGH, a.a.O. (s.o. Fn. 4), Rn. 28; Breucker, a.a.O. (s.o. Fn. 6), 133.

100 St. Rspr. seit RGZ 63, 379; vertiefend jeweils MünchKomm-Baldus, a.a.O., $\mathbb{S} 1004$, Rn. 134; Pa-

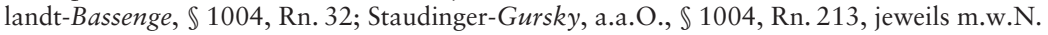

101 BGH, Urt. v. 17.9.2004, Az. V ZR 230/03, NJW 2004, 3701 (st. Rspr. seit BGHZ 2, 394); Palandt-

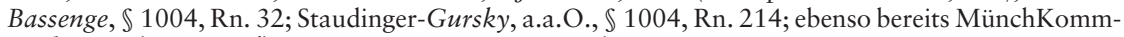
Medicus, Bd. 6, 4. Aufl., 2004, $\mathbb{1} 1004$, Rn. 95, jeweils m.w.N. 
rechtswidrigen Beeinträchtigungen vermutet, kann aber auch bei einer erstmals drohenden Beeinträchtigung gegeben sein. "102

Schon früh hat Breucker klargestellt, dass konkrete Tatsachen vorliegen müssen, auf Grund derer der Schluss gezogen werden kann, dass in Zukunft sicherheitsbeeinträchtigende Handlungen von der betroffenen Person ausgehen werden. ${ }^{103}$ Die bloße Einleitung eines Ermittlungsverfahrens sei hierfür aber in keinem Fall ausreichend. ${ }^{104}$

Jüngst hat Klesczewski zur Ermittlung tauglicher Bezugstatsachen für diese Gefahrprognose zu Recht darauf hingewiesen, dass die Dogmatik des $\mathbb{1 0 0 4}$ BGB an die Terminologie des Polizeirechts anschließt. Mit einem Rückgriff auf das dem Stadionverbot am meisten ähnelnde Aufenthaltsverbot arbeitet er heraus, dass auf äußere Indizien, die einen Rückschluss auf die zu beurteilende innere Gesinnung des Betroffenen zulassen und tragfähig begründen können, abzustellen ist; solche wären beispielsweise das Ankündigen einer Straftat, das Mitsichführen von Tatwerkzeug und einschlägige Vorstrafen. ${ }^{105}$ Das Merkmal der Zugehörigkeit zu einer Gruppe, das i.Ü. in keiner der im Strafrecht anerkannten Prognosetafeln vorkomme, sei, wenn überhaupt, allenfalls ein untergeordnetes Kriterium für eine zukünftige Gewaltbereitschaft. Es sei wenig überzeugend, die Besorgnis einer künftigen Störung auf dieses vereinzelte Merkmal stützen zu wollen. ${ }^{106}$

Wertvoll sind in diesem Zusammenhang die Hinweise von Klesczewski, dass es beim Tatsachenbegriff nicht auf die straf- oder polizeirechtliche Bewertung der zur Verfügung stehenden Sachverhaltsgrundlage, sondern alleine auf den zivilprozessualen Tatsachenbegriff ankommt. Zu Grunde gelegt werden können für die zivilrechtliche Gefahrprognose also alleine unstreitige oder bewiesene Tatsachen.

Neuerdings meinen Walker/Klopp in Reaktion auf die BGH-Entscheidung, dass für eine entsprechende Prognose die Einleitung eines Ermittlungsverfahrens grundsätzlich ausreichend sei. Der Kläger sei Teil einer Gewalt stiftenden Gruppe gewesen, was indiziere, dass er sich beim Fußball im gewaltbereiten Umfeld bewege und deshalb von ihm künftige Störungen zu besorgen seien. Dieser sehr weit gefasste $\mathrm{Zu}-$ schnitt des Adressatenkreises möge zunächst überraschen, finde aber eine Stütze in der Wertung des $\$ 830$ Abs. 1 Satz 2 BGB. Danach hafteten mehrere Beteiligte auch dann gesamtschuldnerisch, wenn sich nicht ermitteln lässt, wer von ihnen den Schaden durch seine Handlung verursacht hat. Ebenso wie dem Geschädigten im Deliktsrecht sei damit dem Fußballverein als Hausrechtsinhaber ein Modus an die Hand

102 BGH, a.a.O. (s.o. Fn. 4), Rn. 17.

103 Breucker, a.a.O. (s.o. Fn. 6), 135; vgl. i.Ü. bereits oben unter B. IV. 2.

104 Breucker, a.a.O.

105 Klesczewski, a.a.O. (s.o. Fn. 5), S. 253.

106 Klesczewski, a.a.O. (s.o. Fn. 5), S. 253. 
gegeben, um effektiv gegen potenzielle oder tatsächliche Störer vorgehen zu können, ohne dabei den Kreis der sanktionierbaren Personen unbillig weit zu ziehen. ${ }^{107}$

Obwohl die Heranziehung dieses Rechtsgedankens zunächst charmant scheint, ist der eingeschlagene Weg nicht zu Ende gedacht und zu kritisieren. Bei diesem Ansatz wird nämlich vernachlässigt, dass sich die gesamtschuldnerische Haftung aller Beteiligten nach $\$ 830$ Abs. 1 Satz 2 BGB eben nicht aus einer bloßen Präsenz bei deliktsrechtlich relevanten Auseinandersetzung ergibt, sondern vielmehr beim potentiellen Mitschädiger nur eintreten kann, wenn insoweit bei ihm nicht unerhebliche subjektive Voraussetzungen vorliegen. Denn vorsätzliches Mitwirken an der Verletzungshandlung gehört zum Tatbestand des $\$ 830$ Abs. 1 Satz 1 BGB, ${ }^{108}$ auf den $\$ 830$ Abs. 1 Satz 2 BGB verweist. Ein sich zufällig in einer gewaltbereiten Gruppe Befindender hat aber einen solchen Vorsatz nicht; auch unternimmt er keine eigene Gefährdungshandlung, die aber Voraussetzung für die Anwendbarkeit von $\mathbb{\$} 830$ Abs. 1 Satz 2 BGB ist. ${ }^{109}$ Dass eine solch substanzlose Gefahrprognose gerade den „Kreis der sanktionierbaren (sic!) Personen“ unbillig erweitert, wird sogleich ausführlich dargelegt.

Heermann meint, dass die Feststellungen des BGH zur zufälligen Gruppenzugehörigkeit den praktischen Bedürfnissen der Veranstalter entgegen kämen. Bedenklich hierbei sei, dass bei unreflektierter, die Besonderheiten des Einzelfalls vernachlässigender Anwendung der SVRL die Einleitung eines Ermittlungsverfahrens durch die Polizei ein Stadionverbot für die Fans gleichsam automatisch nach sich zieht. ${ }^{110}$

\section{c) Auffassung des BGH zur Gefahrprognose beim Stadionverbot}

Der BGH ${ }^{111}$ hält eine Gefahrenprognose auf Grund eines eingeleiteten Ermittlungsverfahrens, das später nach $\$ 153$ StPO eingestellt wurde, für tragfähig.

Der seinerzeit 16-jährige Kläger, der als Anhänger des FC Bayern München am 25. März 2006 das Auswärtsspiel seines Vereins beim MSV Duisburg besuchte, wurde nach Spielschluss als Teil einer Gruppe von ca. 100 Menschen identifiziert, aus welcher heraus tätliche Auseinandersetzungen mit Personen- und Sachschäden verwirklicht wurden. Das daraufhin wegen Landfriedensbruch eingeleitete Ermittlungsverfahren u.a. gegen den Kläger wurde später nach $\mathbb{1 5 3}$ StPO - obwohl $\mathbb{} 45$ JGG die für den jugendlichen Beschuldigten richtige Norm gewesen wäre ${ }^{112}$ - eingestellt, während das zuvor durch den MSV Duisburg verhängte Stadionverbot nach

107 Walker/Klopp, Anmerkung zu BGH, Urt. v. 30.10.2009, LMK 2010, 295984.

108 Allgemeine Ansicht, vgl. statt vieler nur Staudinger-Eberl-Borges, BGB (Neubearbeitung 2008), $\mathbb{\$} 830$, Rn. 12 f.; Palandt-Sprau, $\mathbb{\$} 830$, Rn. 2, jeweils m.w.N.

109 Staudinger-Eberl-Borges, a.a.O., $\$ 830$, Rn. 11; Palandt-Sprau, $\$ 830$, Rndr. 7.

110 Heermann, Anmerkung, NJW 2010, 537.

111 BGH, a.a.O. (s.o. Fn. 4).

112 Vgl. nur Schäfer/Sander/Gemmeren, a.a.O. (s.o. Fn. 60), Rn. 48. 
durch den Kläger beantragter erneuter Überprüfung durch den Verein aufrecht erhalten wurde. ${ }^{113}$ Das bis zum 30. Juni 2008 befristete überörtliche Stadionverbot selbst hatte sich zwar zwischenzeitlich erledigt, jedoch verlor der Kläger wegen des Verbotssausspruchs seine Mitgliedschaft und Dauerkarte beim FC Bayern München, weshalb er - wie auch zutreffend vom BGH bestätigt wurde ${ }^{114}$ - weiterhin ein rechtliches Interesse an der Feststellung der Rechtswidrigkeit des ausgesprochenen Stadionverbots hatte. ${ }^{115}$ Jedoch hielt der BGH alleine wegen der festgestellten Zugehörigkeit des Klägers zu der beschriebenen Gruppe das Stadionverbot für rechtmäßig. Hierzu führt der Senat aus:

„Bei der Festsetzung von Stadionverboten sind andere Maßstäbe anzuwenden als bei der strafrechtlichen Sanktionierung von Störungen bei früheren Spielen. Während insoweit nach dem Grundsatz in dubio pro reo eine Bestrafung unterbleibt, wenn keine Tat bewiesen ist, können Stadionverbote eine nennenswerte präventive Wirkung nur dann erzielen, wenn sie auch gegen solche Besucher ausgesprochen werden, die zwar nicht wegen einer Straftat verurteilt sind, deren bisheriges Verhalten aber besorgen lässt, dass sie bei künftigen Spielen sicherheitsrelevante Störungen verursachen werden. "116

„Der Kläger ist nicht zufällig in die Gruppe, aus der heraus Gewalttaten verübt worden sind, geraten, sondern war Teil dieser Gruppe. Die Zugehörigkeit zu dieser Gruppe, mit der der Kläger in Gewahrsam genommen wurde, rechtfertigt die Annahme, dass er sich bei Fußballveranstaltungen in einem zu Gewalttätigkeiten neigenden Umfeld bewegt und von ihm deshalb künftige, Dritte gefährdende Störungen zu besorgen sind; auf den Nachweis, er habe sich an den aus der Gruppe heraus begangenen Gewalttätigkeiten beteiligt, kommt es [...] nicht an. "117

Mit der BGH-Entscheidung wurde ein für die Ermittlung einer Gefahrenprognose nunmehr vermeintlich entscheidendes Kriterium in den Fokus gerückt: die Zugehö-

113 BGH, a.a.O. (s.o. Fn. 4), Rn. 3.

114 BGH, a.a.O. (s.o. Fn. 4), Rn. 8 ff.

115 Hierzu: Breucker, SpuRt 2010, 31 (letzter Absatz).

116 BGH, a.a.O. (s.o. Fn. 4), Rn. 19 unter Hinweis auf AG Freiburg, SpuRt 2005, 257. Dieser Beleg überrascht und trägt nicht. Hierbei soll nicht diskutiert werden, ob dies schon daran liegt, dass das oberste deutsche Gericht für Zivilsachen seine tragenden Erwägungen mit einer singulären amtsgerichtlichen Entscheidung belegt, die zudem den gezogenen Schluss jedenfalls nicht ausdrücklich enthält. Der Senat hat aber übersehen, dass sich die Entscheidung des Amtsrichters - die darüber hinaus in ihrem pädagogisch-moralisierenden obiter dictum am Schluss der veröffentlichen Entscheidungsgründe einen haut gồt entfaltet, von dem sich der BGH distanzieren sollte - auf eine Fassung der SVRL bezieht, die zwischenzeitlich weit überholt ist. Die Entscheidung des AG Freiburg bezieht sich offensichtlich auf die vor dem 18.2.2005 geltende Fassung der SVRL (noch erlassen auf Grund von \30 der Richtlinien zur Verbesserung der Sicherheit bei Bundesligaspielen), die eine Aufhebung eines ausgesprochenen Stadionverbots nur „bei erwiesener Unschuld“ des Betroffenen vorsah. Hier ist zwischenzeitlich sogar der Richtliniengeber zu Recht schlauer, vgl. oben unter B. III. 2.

117 BGH, a.a.O. (s.o. Fn. 4), Rn. 23. 
rigkeit zu einer Gruppe, aus der heraus Gewalthandlungen verübt wurden. Zugehörigkeit dürfte damit zum Schlüssel zukünftiger Stadionverbotsfestsetzungen erstarken. Seinen neu kreierten terminus technicus versäumt der BGH jedoch mit Inhalt zu füllen, obwohl dieses Tatbestandsmerkmal einige Fragen hinsichtlich seiner objektiven Grundlagen, seiner subjektiven Einflüsse und auch der dadurch ausgelösten Rechtsfolgen aufwirft. ${ }^{118}$

\section{aa) Objektive Grundlagen einer „Zugehörigkeit“}

Wörtlich versteht man unter „Zugehörigkeit“ das bloße „Dazugehören“, 119 also die wertneutrale, auf objektiven Tatsachen gestützte Feststellung, Teil eines Ganzen zu sein. ${ }^{120}$ Qualitativ grenzt sich die bloße Zugehörigkeit zu einer Gruppe, aus der heraus Gewalttaten verübt worden sind, damit gerade von einer aktiven Beteiligung an festgestellten Gewalthandlungen und insbesondere von der tatbestandlichen Verwirklichung eines Landfriedensbruchs nach $\$ 125 \mathrm{StGB}$ ab. ${ }^{121}$ Es genügt schon, sich lediglich im räumlichen Umfeld gewalttätiger Personen aufzuhalten.

Auch in zeitlicher Hinsicht ist das Merkmal der Zugehörigkeit äußerst schnell erfüllt. Der BGH bezieht nämlich keine Stellung zu einer möglichen Differenzierung dahingehend, ob die Zugehörigkeit zu einer Gruppe der beschriebenen Art bereits vor der ersten erkennbaren Gewalthandlung oder erst danach begründet wurde.

Hinzu kommt schließlich, dass nach allgemeiner Lebenserfahrung sich insbesondere die Anhänger der Gastmannschaft zum Zwecke der Vereinfachung der Auswärtsfahrt regelmäßig entweder organisiert oder spontan zu Gruppen mit persönlich unbekannter Zusammensetzung zusammenschließen oder sogar von den örtlichen Sicherheitskräften zwangsweise zusammengeschlossen werden. ${ }^{122}$ Organisierte Fahrten zu Auswärtsspielen in dafür angemieteten Bussen oder Sonderzügen sind ebenso alltäglich wie die einhergehende Ungewissheit, ob sich innerhalb solcher Zweckgemeinschaften überwiegend miteinander unbekannter Personen auch gewaltbereite Menschen aufhalten. Gleiches gilt für spontane Zusammenschlüsse z.B. am Hauptbahnhof des Gastspielortes, um gemeinsam zum Stadion weiterzureisen oder von dort wieder zum Bahnhof zurückzukehren. Diese unter Fans üblichen Solidarisierungen geschehen in der Regel, um sich in einer fremden Umgebung in der Gruppe besser zurecht zu finden und womöglich auch - und man muss sagen: leider - sich

118 Sehr kritisch insoweit auch, wenn auch mit anderer Schwerpunktsetzung, Klesczewski, a.a.O. (s.o. Fn. 5), S. $252 \mathrm{ff}$.

119 Duden, Deutsches Universalwörterbuch (6. Aufl. 2006), S. 1989.

120 Dies kann sogar die bloße Mitgliedschaft in einem Fanclub betreffen, vgl. dazu das Beispiel der „Münchener Schickeria“ bei Klesczewski, a.a.O. (s.o. Fn. 5), S. 252.

121 Während $\mathbb{} 125$ StGB a.F. die Zugehörigkeit zu einer gewalttätigen Gruppe ausdrücklich umfasste und unter Strafe stellte, gilt dies seit dem 3. StrRG vom 20.5.1970 gerade nicht mehr, vgl. statt vieler Schönke-Schröder-Lenckner/Sternberg-Lieben, StGB-Kommentar (27. Aufl. 2006), \125, Rn. 1, 12.

122 Ebenso - mit sehr deutlichen Worten - Klesczewski, a.a.O. (s.o. Fn. 5), S. 253. 
vor denkbaren Übergriffen von Anhängern der gegnerischen Mannschaft auf Einzelne zu schützen. Auch hier ist eine mögliche spätere Gewalthandlung von Gruppenmitgliedern nicht vorherzusehen. Erst recht gilt dies für Zwangsgruppierungen, wie sie besonders durch örtliche Polizei- und Ordnungskräfte an Fans der Gastmannschaft vorgenommen werden, sei es durch physische Trennung des Gästebereichs im Stadion oder durch Trennung und Kanalisierung der Fangruppen vor und nach dem Spiel in Form von Einkesselungen durch Hundertschaften bis hin zur organisierten Polizeieskorte von Pendelbussen zwischen Stadion und Hauptbahnhof. Der Einzelne kann sich solchen Maßnahmen nicht entziehen, obwohl sich bei einem als überhart empfundenen Polizeieinsatz gerade innerhalb solcher Zwangsgruppierungen einige Anhänger leicht zu auch gruppendynamischen Gewalthandlungen provozieren lassen können.

\section{bb) Subjektive Einflüsse auf Zugehörigkeit}

Besonders die letzten Überlegungen leiten zu den subjektiven Einflüssen der Zugehörigkeit über. Wie sich anhand der lebensnahen Beispiele leicht erschließen lässt, ist ein auf Zugehörigkeit zu einer gewaltbereiten Gruppe gerichteter Vorsatz nicht notwendig. Während im Kontrast dazu zur Verwirklichung von $\$ 125 \mathrm{StGB}$ Gewalthandlungen vorsätzlich ausgeübt werden müssen und reine Unterstützungstätigkeiten sogar Absicht erfordern, ${ }^{123}$ fehlen solche qualifiziert subjektiven Anforderungen bei der Frage der Zugehörigkeit völlig. Der BGH differenziert lediglich nach dem Kriterium der „Zufälligkeit“; wer zufällig in eine solche Gruppe gerät, soll nicht als zugehörig gelten. ${ }^{124}$ Wie soeben gesehen, hält man sich als Fußballfan jedoch in der Regel nie lediglich zufällig in einer Gruppe anderer Anhänger desselben Vereins auf. Vom Zufall hängt es hingegen sehr häufig ab, ob von der jeweiligen Gruppe (später) auch Gewalt ausgeht. Dann ist die Zugehörigkeit jedoch schon unzufällig begründet worden, sodass der BGH in solchen praktisch immer einschlägigen Fällen eine Zugehörigkeit bejahen müsste.

\section{cc) Rechtsfolgen einer Zugehörigkeit}

Ist einmal eine Zugehörigkeit zu einer Gruppe, aus welcher heraus Gewalttaten verübt worden sind, festgestellt, so kann dies als strafrechtlich relevante Rechtsfolge allenfalls einen Anfangsverdacht für Ermittlungsverfahren begründen; andernfalls wären Tatsachenfeststellungen zu $\mathbb{1 2 5} \mathrm{StGB}$ auch nur schwerlich möglich. Erst im Rahmen des Ermittlungsverfahrens kann dann festgestellt werden, ob der jeweilige einzelne Gruppenzugehörige tatsächlich auch Gewalt angewendet oder zumindest absichtlich Unterstützungsbeiträge dazu geleistet hat. 
Daraus folgt aber auch, dass mit der bloßen Zugehörigkeit zu einer Gruppe die Gefahrprognose für ein Stadionverbot (s.o. C. I. 4.) nicht begründet werden kann.

\section{d) Zwischenergebnis}

Taugliche Maßstäbe einer Gefahrenprognose sind allein die anerkannten Merkmale aus den straf- und polizeirechtlichen Prognosetafeln, die in Abwägung mit der Persönlichkeit des Betroffenen unter Berücksichtigung der Besonderheiten des Stadionverbots die Besorgnis tragen müssen, dass von dem Betroffenen in Zukunft weitere sicherheitsrelevante Störungen ausgehen werden. Die bloße Zugehörigkeit zu einer Gruppe, aus welcher heraus Gewalthandlungen verübt wurden, kann hingegen für sich genommen niemals eine verlässliche Gefahrenprognose begründen. Ebenso wenig kann die Einleitung eines Ermittlungsverfahrens oder die Einstellung eines solchen nach $\mathbb{S} 153$ StPO diese Prognose tragfähig begründen. Das gleiche gilt für die Biografie des Betroffenen. Sie allein genügt nicht für die sichere Annahme, der Betroffene werde auch in Zukunft in gleicher Weise die öffentliche Sicherheit stören. ${ }^{125}$

\section{Anspruch aus $\S 862$ Abs. 1 Satz 2 BGB}

Der zur Durchsetzung eines Stadionverbots notwendige Unterlassungsanspruch kann sich aber auch aus den besitzrechtlichen Vorschriften des BGB ergeben. So gewährt $\int 862$ Abs. 1 Satz 2 BGB einen entsprechenden Unterlassungsanspruch.

\section{Beeinträchtigung von „Besitz“ (possessorisch)}

Der Besitz i.S.v. $\$ 854$ Abs. 1 BGB, auch der gegenüber dem Störenden möglicherweise unberechtigte und sogar der fehlerhafte, ${ }^{126}$ ist durch ausschnittsweisen Entzug der durch ihn gewährleisteten Gebrauchs- und Nutzungsmöglichkeit gestört. ${ }^{127}$ Eine Besitzstörung ist damit unter den gleichen Voraussetzungen anzunehmen, unter welchen auch eine Beeinträchtigung i.S.d. $\mathbb{1} 1004$ BGB vorliegt. Insoweit kann nach oben verwiesen werden (s.o. unter C. I. 1.).

\section{Wiederholungsgefahr}

Die für den Unterlassungsanspruch nach $\$ 862$ Abs. 1 Satz 2 BGB erforderliche Wiederholungsgefahr ist mit der für $\$ 1004$ Abs. 1 Satz 2 BGB notwendigen Wiederholungsgefahr identisch (s.o. unter C. I. 4). Somit gelten insoweit für beide Ansprüche die gleichen Voraussetzungen.

125 OLG Köln, Urt. v. 17.1.2007, Az. 16 Wx 220/06, SpuRt 2007, 208.

126 MünchKomm-Joost, Bd. 6, 5. Aufl., 2009, $\$ 863$, Rn. 6; Palandt-Bassenge, $\$ 862$, Rn. 7.

127 Staudinger-Bund, BGB (Neubearbeitung 2007), $\mathbb{8} 862$, Rn. 7; Palandt-Bassenge, $\mathbb{8} 862$, Rn. 2; MünchKomm-Joost, a.a.O., $\$ 862$, Rn. 1 . 


\section{Verbotene Eigenmacht}

Diese Besitzstörung hat durch verbotene Eigenmacht zu erfolgen, $\mathbb{\int} 862$ Abs. 1 Satz 1 BGB. Verbotene Eigenmacht ist legal definiert in $\$ 858$ Abs. 1 BGB. Gesetzliche Rechtfertigungsgründe für eine Besitzstörung im Zusammenhang mit Stadionbesuchen sind, von Notfällen abgesehen, nicht ersichtlich, sodass dieses Merkmal regelmäßig erfüllt sein wird.

\section{Konkurrenzen}

Die Anspruchskonkurrenz zu den bereits dargestellten Unterlassungsansprüchen nach $\int 1004$ Abs. 1 Satz 2 BGB ist unproblematisch. Die Ansprüche bestehen kumulativ. ${ }^{128}$

\section{Grenzen, insbesondere $§ 864$ BGB}

Der Unterlassungsanspruch nach $\ 862$ Abs. 1 Satz 2 BGB unterliegt allerdings einer wichtigen Einschränkung. Er erlischt gemäß $\ 864$ Abs. 1 BGB nach Ablauf eines Jahres nach Verübung der verbotenen Eigenmacht, wenn der Anspruch nicht vorher im Wege der Klage geltend gemacht wird. Da die Vereine statt einer Klageerhebung immer den effektiven und kostengünstigen Weg wählen werden, das Stadionverbot dadurch durchzusetzen, dass die Betroffenen durch die Sicherheitskräfte schlicht nicht ins Stadion hineingelassen werden, unterliegen alle auf der Grundlage von \862 Abs. 1 Satz 2 BGB ergangenen Stadionverbote einer Verfallsfrist von einem Jahr seit der ihre Aussprache begründenden Störung.

\section{Zwischenergebnis}

Stadionverbote können auf die zivilrechtlichen Unterlassungsansprüche der $\$ 1004$ Abs. 1 Satz 2 BGB (unmittelbar für Stadieneigentümer), $\mathbb{S} \mathbb{S} 1004$ Abs. 1 Satz 2 analog, 823 Abs. 1 BGB (für berechtigte Stadienbesitzer) und $\$ 862$ Abs. 1 Satz 2 BGB (direkt für alle Stadienbesitzer) gestützt werden. Anspruchsgrundlage der Wahl für die Praxis muss $\mathbb{S} \int 1004$ Abs. 1 Satz 2 analog, 823 Abs. 1 BGB bleiben, weil die Gesellschaften der Ligen regelmäßig nicht Eigentümer, aber natürlich berichtigte Besitzer der Stadien sind und nur so eine a priori Befristung der Stadionverbote von einem Jahr ( $\int 864$ Abs. 1 BGB) vermieden werden kann. ${ }^{129}$

128 Staudinger-Bund, a.a.O., $\$$ 862, Rn. 2; Palandt-Bassenge, $\mathbb{8} 862$, Rn. 1; MünchKomm-Joost, a.a.O., \$ $862, \mathrm{Rn} .1$.

129 Für diesen vorzugswürdigen Weg sprachen sich auch schon Breucker, a.a.O. (s.o. Fn. 6) 136 und Orth, a.a.O. (s.o. Fn. 6), S. 139., S.137, aus. Dagegen hält der BGH, a.a.O. (s.o. Fn. 4), Rn. 16, $\int 862$ BGB für einschlägig und geht auf den Schutz des berechtigten Besitzes überhaupt nicht ein. Vor diesem Hintergrund überrascht die Urteilsanmerkung Breuckers, SpuRt 2010, 31 (Punkt 1), welche dem BGH eine Klarstellung „in erfrischender Deutlichkeit“ attestiert. Siehe zu dieser in diesem Fall durchaus brenzligen Problematik ferner unten unter D. III. 2. 


\section{Nebenansprüche aus Besitzstörung}

Die besitzrechtlichen Nebenansprüche des Hausrechtsinhabers gegen einen störenden Fan aus seiner verbotenen Eigenmacht, $\mathbb{S} \mathbb{S} 858$ Abs. 1, 859 Abs. 2, 860 BGB geben zudem die Möglichkeit, ein Stadionverbot auf Grund eines akuten Vorfalls sofort vor Ort umzusetzen. ${ }^{130}$

\section{Einfluss des Verfassungsrechts}

Neben seinem zivilrechtlich hergeleiteten Fundament wohnt jedem ausgesprochenen Stadionverbot ferner eine verfassungsrechtliche Dimension inne. Denn auch wenn das Rechtsverhältnis zwischen dem Stadionverbotsfestsetzer und dem betroffenen Fan rein privatrechtlicher Natur ist, so werden dadurch allein noch keine grundrechtlichen Garantien ausgeschlossen. Es gilt daher, die auf Grund eines Stadionverbots denkbaren Grundrechtsbeeinträchtigungen in rein privatrechtlichen Beziehungen genauer zu überprüfen.

\section{Wirkung von Grundrechten in rein privatrechtlichen Beziehungen}

Unbestritten ist heute, dass Grundrechte jedenfalls nicht direkt auf rein privatrechtliche Beziehungen anzuwenden sind. ${ }^{131}$ Jedoch entfalten sie grundsätzlich auch auf solche Rechtsverhältnisse einen nicht unerheblichen Einfluss über oft als „,(mittelbare) Drittwirkung“ oder „Horizontalwirkung“ bezeichnete, in Einzelheiten umstrittene Konstruktionen. ${ }^{132}$ Die im Ergebnis erzeugte Ausstrahlungswirkung ${ }^{133}$ der Grundrechte auf das Privatrecht führt letztlich doch zu einem mittelbaren Einfluss einzelner Grundrechte auf privatrechtliche Rechtsverhältnisse. ${ }^{134}$ Dies wird u.a. mittels verfassungskonformer Auslegung einfachgesetzlicher Normen, jedenfalls und insbesondere der Generalklauseln des Zivilrechts, verwirklicht. ${ }^{135}$ Die verfassungskonforme Anwendung des einfachen Rechts wird durch die Zivilgerichte, die als Teil der staatlichen Gewalt gem. Art. 1 Abs. 3 GG unmittelbar an die Grundrechte gebunden sind, verbürgt. ${ }^{136}$ Daher haben sämtliche ordentliche Gerichte, die über die

130 Breucker, a.a.O. (s.o. Fn. 6), S. 134; Orth, a.a.O. (s.o. Fn. 6), S. 137

131 Ipsen, Staatsrecht II, 11. Aufl. 2008, Rn. 68ff.; Pieroth/Schlink, Staatsrecht II, 24. Aufl. 2008, Rn. 173 ff.; vgl. i.Ü. instruktiv Canaris, Grundrechte und Privatrecht, Schriftenreihe der Juristischen Gesellschaft zu Berlin, Heft 159 (1999).

132 Umfassend dazu Stern, Staatsrecht III/1, \ 76, S. 1511 ff., sowie aus der neueren Literatur jeweils m.w.N. Höfling, in: Sachs, GG, 5. Aufl. 2009, Art. 1, Rn. 111 f.; Schmidt-Bleibtreu/Hofmann/Hopfauf-Kannengießer, GG, 11. Aufl. 2008, Vorb. v. Art. 1, Rn. 6 f.

133 Sachs, in: ders., GG, 5. Aufl. 2009, Vor Art. 1, Rn. 32 m.w.N.; über den Begriff lässt sich indes trefflich streiten, vgl. nur Canaris, a.a.O. (s.o. Fn. 131), S. $30 \mathrm{ff.}$

134 Grundlegend seit BVerfGE 7, 198 („Lüth“), vgl. dazu nur Stern, a.a.O. (s.o. Fn. 132), S. 1547 m.w.N.

135 Sack, in: Staudinger, BGB, $\mathbb{} 134$ (Neubearbeitung 2003), Rn. 41; weiterführend Stern, a.a.O. (s.o. Fn. 132), S. $1556 \mathrm{ff}$.

136 Canaris, a.a.O. (s.o. Fn. 131), S. 23 ff. m.w.N. 
Rechtmäßigkeit eines festgesetzten Stadionverbots zu befinden haben, dabei stets die Grundrechtspositionen der Verfahrensbeteiligten zu berücksichtigen. ${ }^{137}$

Beim Streit über die Zulässigkeit eines verhängten Stadionverbots finden die Grundrechte des Betroffenen jedenfalls durch die Generalklausel des $\int 826$ BGB Eingang in das Verfahren; der Betroffene sieht sich nämlich regelmäßig dann einer sittenwidrigen Schädigung ausgesetzt, wenn das Stadionverbot ohne sachlichen Grund in eine geschützte Grundrechtsposition eingreift. Dabei ist von besonderer Bedeutung, dass grundsätzlich zwischen Stadionbetreiber und (potentiellem) Stadionbesucher ein Kontrahierungszwang gerichtet auf einen Stadionbesuchsvertrag besteht. ${ }^{138}$ Beide Grundrechtsträger befinden sich folglich bereits im Ausgangspunkt in einem asymmetrischen Verhältnis dergestalt, dass die Ausübung der Privatautonomie durch den vom Kontrahierungszwang begünstigten Fan im Zweifel Vorrang genießt. Im Streitfall haben die Zivilgerichte seine Grundrechtspositionen stärker zu gewichten.

Damit entfalten die Grundrechte eines von einem Stadionverbot Betroffenen dadurch Wirkung, dass der Zivilrichter verpflichtet ist, ihrer Schutzfunktion ${ }^{139}$ Geltung zu verschaffen.

\section{Grundrechtsbeeinträchtigungen in concreto}

Im Einzelnen könnten durch die Aussprache eines Stadionverbots folgende Grundrechte verletzt sein:

\section{Art. 11 Abs. $1 \mathrm{GG}$ - Freizügigkeit}

Zunächst könnte die Festsetzung eines Stadionverbots gegen das Grundrecht der Freizügigkeit aus Art. 11 Abs. 1 GG verstoßen, ${ }^{140}$ soweit dies zum Nachteil eines Deutschen oder eines EU-Bürgers geschieht. ${ }^{141}$ Die sich um die Anwendung von Art. 11 GG auf das Stadionverbot scharenden Probleme sind vielfältig und oftmals wenig ausdiskutiert. Die folgenden Ausführungen beanspruchen daher auch nicht Probleme zu lösen, sondern sie für zukünftige Diskussionen offenzulegen.

Freizügigkeit verbürgt die freie Aufenthalts- und Wohnsitznahme innerhalb der Bundesrepublik und umfasst dabei auch die zu diesem Zwecke notwendige Fortbewegung. ${ }^{142}$ Ein Stadionverbot kann allenfalls in die Aufenthaltsnahme eingreifen, so-

137 Von unveränderter Aktualität Doehring, Das Staatsrecht der Bundesrepublik Deutschland, 3. Aufl. 1984, S. 209: „Das Gericht hat die Grundrechte zu beachten, soweit sie gelten; nicht etwa gelten sie, weil ein Gericht entscheidet."

138 S.o. A. II; dies begründet hier i.Ü. auch die besondere Schutzfunktion der Grundrechte innerhalb des Zivilrechts, Canaris, a.a.O. (s.o. Fn. 131), S. 80 ff.

139 Zur Herleitung der Schutzfunktion überzeugend Canaris, a.a.O. (s.o. Fn. 131), S. 38 f.

140 So in rechtsvergleichendem Kontext auch Klesczewski, a.a.O. (s.o. Fn. 5), S. 253.

141 Zum personalen Schutzbereich von Art. 11 GG s. statt vieler Schmidt-Bleibtreu/Hofmann/HopfaufHofmann, Grundgesetz Kommentar, 11. Aufl. 2008, Art. 11, Rn. 3 ff.

142 Sachs-Pagenkopf, Grundgesetz Kommentar, 5. Aufl. 2009, Rn. 14. 
weit man entgegen der Auffassung der Verfasser das Verweilen in einem Stadion während eines Fußballspiels bereits als Aufenthalt im grundrechtlichen Sinne verstehen möchte. ${ }^{143}$ Zweifelhaft ist ferner, ob speziell Art. 11 Abs. 1 GG überhaupt in rein privatrechtlichen Beziehungen anwendbar sein kann, ${ }^{144}$ weil er ersichtlich an rein hoheitliche Lebenssachverhalte adressiert ist und der qualifizierte, besonders detailliert ausgestaltete Gesetzesvorbehalt von Art. 11 Abs. 2 GG dem Wortlaut nach nur auf Staat-Bürger-Konstellationen zurechtgeschnitten ist. ${ }^{145}$ Ferner ist zuletzt fraglich, ob dem qualifizierten Gesetzesvorbehalt nicht schon durch die angesprochenen Vorschriften des BGB (freilich unter Missachtung des Zitiergebots von Art. 19 Abs. 4 GG) Genüge getan wurde. Wäre dies der Fall, so gälte es nur noch die Anwendung des Gesetzes zu prüfen, was konstruktiv und argumentativ den sogleich folgenden Ausführungen zu Art. 2 GG entspräche, auf welche dann verwiesen werden soll.

Dessen ungeachtet erscheint es nicht nur angesichts der bestehenden Rechtsunsicherheit, sondern auch der allenfalls vom Zufall - nämlich der Nationalität des von einem Stadionverbot Betroffenen - abhängenden Schutzbereichseröffnung im Wechsel zwischen Freizügigkeit und Allgemeinem Persönlichkeitsrecht wenig sachgerecht, Art. 11 GG hier überhaupt heranzuziehen.

\section{Art. 2 Abs. 1 i.V.m. Art. 1 Abs. 1 GG - Allgemeines Persönlichkeitsrecht}

Eine Verletzung von Art. 2 Abs. 1 i.V.m. Art. 1 Abs. 1 GG liegt vor, wenn ein Eingriff in den Schutzbereich des Allgemeinen Persönlichkeitsrechts des Betroffenen vorliegt, der verfassungsrechtlich nicht gerechtfertigt ist. ${ }^{146}$ Auf unterster Stufe der hierzu entwickelten Sphärentheorie schützt das Allgemeine Persönlichkeitsrecht auch die

143 Die Definition der Aufenthaltsnahme ist stark umstritten; zuweilen wird als Abgrenzung zur bloßen Bewegungsfreiheit, die unter den Schutzbereich von Art. 2 GG fällt, für den Aufenthalt eine gewisse zeitliche Dauer, ein Ortswechsel von einiger Bedeutung und Dauer oder aber jedenfalls ein Ortswechsel mit eingeschlossener Übernachtung gefordert, vgl. zum Streitstand jeweils m.w.N. SchmidtBleibtreu/Hofmann/Hopfauf-Hofmann, a.a.O., Art. 11, Rn. 8; Pagenkopf, in: Sachs, a.a.O., Art. 11, Rn. 16; Pieroth/Schlink, a.a.O., Rn. 791. Noch weitergehend könnte man wiederum spitzfindig zwischen kurzen Auswärtsfahrten zu „Lokalderbys“ (dann keine erhebliche Dauer, also Art. 2 GG) und Fahrten mit Übernachtung zu Gegnern am anderen Ende des Bundesgebiets (dann erhebliche Dauer, also Art. 11 GG) unterscheiden, was den grundsätzlichen Schutzbereich von Art. 11 Abs. 1 GG wohl kaum tangieren kann. Weiterhin überzeugt mehr, Aufenthalt systematisch als Minus von Wohnsitz zu betrachten und darunter angesichts dessen jedenfalls mehr als das Verweilen bei einer zeitlich eng begrenzten Veranstaltung zu verstehen.

144 Jedenfalls für Konstellationen außerhalb individueller sittenwidriger Vereinbarungen, vgl. dazu $\mathrm{C} a$ naris, a.a.O. (s.o. Fn. 131), S. 72.

145 Gegen eine Drittwirkung von Art. 11 GG scheint sich auch das BVerfG in BVerfGE 80, 137, 150 ausgesprochen zu haben: „Freizügigkeit bedeutet, ungehindert durch staatliche Gewalt (...) Aufenthalt und Wohnsitz nehmen zu können.“ (Hervorh. durch die Verf.) Im bisherigen wissenschaftlichen Diskurs scheint diese Problematik ausgeblendet zu sein, denn damit befasste sich thematisch zuletzt in seiner Habilitationsschrift Ziekow, Über Freizügigkeit und Aufenthalt, Tübingen 1997, S. 572 ff., ohne jedoch inhaltlich speziell dazu etwas beizutragen.

146 Statt vieler Schmidt-Bleibtreu/Hofmann/Hopfauf-Hofmann, a.a.O., Art. 1, Rn. 58 ff., Art. 2, Rn. 22 ff.; Sachs-Murswiek, a.a.O., Art. 2, Rn. 59 ff. 
Sozialsphäre, also die gesamte (Dar-)Stellung eines Menschen nach außen in der Gesellschaft. ${ }^{147}$ In diesen Schutzbereich greift ein festgesetztes Stadionverbot - und damit ein dieses Verbot bestätigendes (höchstinstanzliches) Urteil - ein, wenn es einem Betroffenen damit faktisch die einzige Möglichkeit nimmt, an seiner favorisierten Freizeitbeschäftigung teilzunehmen. Darüber hinaus wird er gegenüber den anderen Fans als potentieller Gewalttäter stigmatisiert und hat ggf. Folgebeeinträchtigungen zu befürchten. ${ }^{148}$ Es stellt einen jedenfalls mittelbaren staatlichen Eingriff dar, wenn eine Gerichtsentscheidung diese Beschränkung aufrechterhält.

Dieser Eingriff kann hier nur mit der verfassungsmäßigen Ordnung gerechtfertigt werden, also der Gesamtheit aller Normen, die formell und materiell mit der Verfassung im Einklang stehen. ${ }^{149}$ Dazu gehören auch das zivilrechtlich garantierte Hausrecht aller Stadionbesitzer, nicht aber eine innerverbandliche Regelung wie die SVRL. Das Hausrecht unterliegt in seiner Anwendung jedoch wie jede gesetzliche Norm dem Verhältnismäßigkeitsgrundsatz als Schranken-Schranke nach der Maßgabe einer an der Eingriffsintensität orientierten Abwägung. ${ }^{150}$ Auf die Wahrung der Verhältnismäßigkeit kommt es also im vorliegenden Fall entscheidend an.

Die dem bundesweiten Stadionverbot zu Grunde liegende Ausübung des Hausrechts eines Bundesligavereins auch in Stellvertretung für sämtliche anderen Vereine im DFB hat wie das Stadionverbot selbst den legitimen Zweck, potenzielle Gewalttäter von Fußballsportveranstaltungen fernzuhalten. Hinsichtlich des Mittels zur Verwirklichung dieses Zwecks ist sodann zu unterscheiden zwischen der Festsetzung des Stadionverbots im Besonderen und der vorausgegangenen Sachverhaltsermittlung zur Begründung eines für die Ausübung des Hausrechts wegen der hier mittelbaren Grundrechtsbindung der Veranstalter notwendigen Gefahrenprognose im Allgemeinen.

\section{a) Die praxisübliche Ausübung des Hausrechts als geeignetes Mittel}

Die Ausübung des Hausrechts ist nur dann ein geeignetes Mittel, wenn sie überhaupt auf den ihr zu Grunde liegenden Sachverhalt passt. Voraussetzung dafür ist eine hinreichend konkrete Ermittlung des Sachverhalts mit daran anknüpfender aus ex anteSicht zutreffender Gefahrenprognose.

Jedenfalls nicht ausreichend ist die - nun durch die BGH-Entscheidung noch stärker in die öffentliche Wahrnehmung als geltendes Recht kolportierte - Bezugnahme zu

147 Hierzu näher Maunz/Dürig-DiFabio, Art. 2 Abs. 1, Rn. 157 ff.

148 Ebenso erging es dem Kläger in der zitierten BGH-Entscheidung, der Dauerkarte und Mitgliedschaft bei seinem Lieblingsverein verlor.

149 Schmidt-Bleibtreu/Hofmann/Hopfauf-Hofmann, a.a.O., Art. 2, Rn. 7 ff.; Sachs-Murswiek, a.a.O., Art. 2, Rn. 89 ff.; Pieroth/Schlink, a.a.O., Rn. 383.

150 Schmidt-Bleibtreu/Hofmann/Hopfauf-Hofmann, a.a.O., Art.2, Rn. 12; Sachs-Murswiek, a.a.O., Art. 2, Rn. 21; Pieroth/Schlink, a.a.O., Rn. 384. 
einer reinen Zugehörigkeit des Betroffenen zu einer Gruppe, aus welcher heraus Gewalthandlungen verübt wurden. Ebenso wenig genügt die unsubstanziierte Feststellung, eine solche Zugehörigkeit sei „nicht zufällig“ erfolgt. Wie gezeigt, kann eine bloße Zugehörigkeit allein niemals eine auch nur in Ansätzen verlässliche Gefahrenprognose begründen. Dazu geeignet sind im Gegenteil erst weitere Erkenntnisse aus dem eingeleiteten staatsanwaltlichen Ermittlungsverfahren. Solche ergeben sich nicht, wenn aus der Aktenlage nur klar war, dass Gewalt aus der Gruppe heraus verübt wurde und sodann das Verfahren aus ermittlungsökonomischen Gründen nach $\$ 153$ anstatt $\$ 170$ Abs. 2 StPO eingestellt wird. Angesichts der staatsanwaltlichen Einstellungspraxis kann dieser Einstellungsgrund ebenfalls nicht als Grundlage einer Gefahrenprognose dienen.

Genau dies greifen aber die SVRL auf und ordnen neben der bereits vorschnell auferlegten Festsetzung eines Stadionverbots bei Einleitung eines Ermittlungsverfahrens bei späterer Verfahrenseinstellung lediglich eine Überprüfung des Verbots an. Strikt nach Vorgabe der SVRL handeln die ihr unterworfenen Vereine und gelangen dann reflexartig zur einer in solchen Fällen üblichen Aufrechterhaltung des Verbots. Eigene Sachverhaltsaufklärung über die staatsanwaltlichen Ermittlungsergebnisse hinaus wird regelmäßig nicht betrieben. Diese branchenübliche sklavische Orientierung an den SVRL ist aber ungeeignet für die Erstellung einer eigenen Gefahrenprognose zur Durchsetzung des Hausrechts. Die SVRL sind mangels Bestandteil der verfassungsmäßigen Ordnung nicht einmal eine taugliche Eingriffsrechtfertigung; gleichwohl werden sie durch sture Befolgung eines Vereins ohne weiteres Zutun genau dazu erhoben. ${ }^{151}$ Auch dies führt zu einer unbrauchbaren Gefahrenprognose und damit zur Ungeeignetheit der Hausrechtsausübung in den beschriebenen praxisüblichen Fällen.

\section{b) Das Stadionverbot in der Mittel-Zweck-Relation}

Daneben ist ferner auf das Stadionverbot als solches einzugehen. Das bundesweite Stadionverbot ist grundsätzlich ein geeignetes und erforderliches Mittel zur Gewaltprävention. Insbesondere existiert kein milderes gleichwirksames Mittel in Form der Aussprache eines örtlichen Stadionverbots, weil so der Zweck der Gewaltprävention angesichts des wöchentlichen Wechsels zwischen Heim- und Auswärtsspielen eines jeden Bundesligavereins nicht gewahrt werden könnte. Es fragt sich aber, ob die Aussprache des Stadionverbots im Einzelfall auch angemessen war, also vor allem ob zwischen Eingriffsintensität und -wirkung hinreichend abgewogen wurde. Die durch das Stadionverbot erlittenen Eingriffe sind gerade für einen Fußballfan besonders einschneidend, auch im Hinblick auf seine soziale Stellung in dem von ihm be-

151 Wie bereits ausgeführt, ist eine auf Tatsachen beruhende, individuelle Gefahrprognose ohne sklavische Betrachtung der SVRL durch den aussprechenden Verein immer möglich und vorzugswürdig, s.o. unter B. II. 2. 
wusst und zulässigerweise (Art. 2 Abs. 1 GG) gewählten gesellschaftlichen Teilbereich. Dagegen erfolgt der Ausspruch eines Verbots üblicherweise nur auf Grund der festgestellten bloßen Zugehörigkeit des Klägers zu einer Gruppe, aus der heraus Gewalthandlungen verübt wurden. Selbst wenn man vor diesem Hintergrund die Sachverhaltsermittlung entgegen aller üblicherweise vorhandenen Fakten für ausreichend und damit die Ausübung des Hausrechts für zumindest geeignet halten möchte, kann das in diesem Rahmen Dargestellte jedenfalls nicht mehr einer Abwägung mit den beeinträchtigten Interessen des Betroffenen standhalten und mündet somit in einem groben Missverhältnis zu Lasten des Betroffenen.

\section{Art. 3 Abs. $1 \mathrm{GG}-$ Willkürverbot}

Ein Verstoß gegen das Willkürverbot aus Art. 3 Abs. 1 GG lässt sich in zweifacher Hinsicht herleiten, nämlich in Bezug auf die Festsetzung eines Stadionverbots und eine diese bestätigende (höchstinstanzliche) Entscheidung direkt sowie hinsichtlich einer Fortsetzung staatlicher Willkür durch Befolgung der SVRL.

\section{a) Stadionverbotsfestsetzung und bestätigende Entscheidung direkt}

Wie bereits dargestellt, stützen sich die praxisübliche Aussprache und Aufrechterhaltung des Stadionverbots wie auch eine bestätigende Gerichtsentscheidung allein auf die „nicht zufällige“ Zugehörigkeit des Betroffenen zu einer Gruppe, aus welcher heraus Gewalthandlungen verübt wurden. Neben dem bereits Festgestellten ist das alleinige Bezugskriterium der Zugehörigkeit sachfremd, weil es keine Erkenntnisse über eine ernsthafte Gefahrenprognose liefern kann. Wenn aber aus sachfremden Erwägungen heraus eine Ungleichbehandlung erfolgt - hier die Verhängung eines Stadionverbots gegen einen betroffenen Gruppenzugehörigen im Vergleich zu nicht belangten Anhängern -, ist dies willkürlich. ${ }^{152}$ Damit würde eine so begründete Festsetzung eines Stadionverbots wie auch ein diese bestätigendes Urteil gegen Art. 3 Abs. 1 GG verstoßen.

\section{b) Fortsetzung staatlicher Willkür}

Aus der staatsanwaltschaftlichen Sicht ist die praktische Bedeutung der Frage, ob das Ermittlungsverfahren gegen einen Beschuldigten im Zusammenhang mit Gewaltausschreitungen in einem Stadion nach $\mathbb{\$} 170$ Abs. 2 oder $\$ 153$ StPO einzustellen ist, gleich null. ${ }^{153}$ Dem Beschuldigten steht gegen beide Einstellungen zudem kein Rechtsmittel zu. ${ }^{154}$ Die Sache erledigt sich, weil auch die möglicherweise anzeigenden Vereine in diesen Grenzfällen kein übermäßiges Verfolgungsinteresse an den Tag legen werden oder im Fall des $\$ 153$ StPO kein eigenes Beschwerderecht haben.

152 Statt vieler Schmidt-Bleibtreu/Hofmann/Hopfauf-Kannengießer, a.a.O., Art. 3, Rn. 16; Pieroth/ Schlink, a.a.O., Rn. 438 ff.; ergänzend ausführlich Sachs-Osterloh, a.a.O., Art. 3, Rn. 8 ff.

153 S. oben unter B. IV. 3. a).

154 S. oben unter B. IV. 3. b). 
Das nachvollziehbare Verlangen nach einer schnellen und „bestandskräftigen“ Erledigung mag in der Hektik des Tagesgeschäfts und angesichts des immensen Arbeitsdrucks der Staatsanwaltschaften verständlich sein. Es dürfte aber auch dazu führen, dass eine trennscharfe staatsanwaltliche Entscheidung hinsichtlich der heranzuziehenden Einstellungsnorm einer „leichten und schnellen“ Einstellung nach $\$ 153$ StPO weicht. Dies ist - wenn auch aus der Sicht der jeweiligen Entscheider menschlich nachvollziehbar - bei Lichte und de iure betrachtet nichts Anderes als Willkür i.S.d. Art. 3 Abs. 1 GG. Dies gilt umso mehr, weil nicht nur in Grenzfällen der entscheidende Beamte der Staatsanwaltschaft immer eher zu einer Einstellung nach $\mathbb{1 5 3}$ StPO neigen wird, um das im Falle der Einstellung nach $\$ 170$ Abs. 2 StPO gegebene Beschwerderecht des $\$ 172$ Abs. 1 StPO zu vermeiden. ${ }^{155}$

Zwar wirkt sich diese Praxis mangels strafrechtlicher Beschwer in beiden Einstellungsarten nicht unmittelbar negativ auf den Betroffenen aus. In Anwendung der SVRL jedoch hat diese Entscheidung erhebliche Konsequenzen: Im Falle einer Einstellung nach $\mathbb{1} 153 \mathrm{StPO}$ wird ein Stadionverbot gegen den Betroffenen aufrecht erhalten. Damit ergeht ein Stadionverbot, welches auf Grund von $\$ 6$ Abs. 2 SVRL bestehen bleibt, willkürlich, wenn sich die SVRL gemäß ihres Wortlauts staatsanwaltliche Willkür nachträglich destruktiv zu eigen machen. Damit verstößt jedes so begründete Stadionverbot gegen Art. 3 Abs. 1 GG. Gleiches gilt für eine dies bestätigende Gerichtsentscheidung. ${ }^{156}$

\section{Verfassungsrechtliche Dimensionen der BGH-Entscheidung zum Stadionverbot}

Vor diesem Hintergrund lassen sich auch die verfassungsrechtlichen Dimensionen der BGH-Entscheidung projizieren. Der Beschwerdeführer einer hiergegen erhobenen Verfassungsbeschwerde müsste vor dem BVerfG seine Betroffenheit durch die Verletzung spezifischen Verfassungsrechts ${ }^{157}$ auf Grund der BGH-Entscheidung nachweisen. Dabei genügt es nicht, die in Verbindung mit den SVRL, ihrer Anwendung und den beides bestätigenden instanzgerichtlichen Entscheidungen dargelegten, auch verfassungsrechtlich begründeten Rechtsverletzungen geltend zu machen; diese Prüfung oblag vielmehr allein den zuvor angerufenen Gerichten. Das BVerfG hätte stattdessen zu untersuchen, inwiefern sich der Umgang des BGH mit dem im konkreten Fall ausgesprochenen Stadionverbot unmittelbar verfassungsrechtlich auswirkt, d.h. ob es in Anwendung und Auslegung des einfachen Rechts auf einer

155 Eine etwaige Beschwerde des Verletzten wird vom Sachbearbeiter selten geschätzt, weil sie mit erheblicher weiterer Arbeit und insbesondere mit der Vorlage an den Abteilungsleiter und die Generalstaatsanwaltschaft verbunden ist.

$156 \mathrm{Ob}$ in dem vom BGH entschiedenen Sachverhalt auch eine Entscheidung nach $\mathbb{} 170$ Abs. 2 StPO möglich gewesen wäre, ist nicht festgestellt.

157 Lechner/Zuck, BVerfGG, 5. Aufl. 2006, \$90, Rn. 90 ff.; Pieroth/Schlink, Grundrechte - Staatsrecht II, 25. Aufl. 2009, Rn. 1279. Von dieser Problematik strikt zu trennen ist der bereits erwähnte Themenkomplex der grundrechtlichen Wirkung auf das Privatrecht, vgl. dazu überzeugend Canaris, a. a. O. (s.o. Fn. 131), S. $27 \mathrm{ff}$. 
grundsätzlich unrichtigen Auffassung von Bedeutung und Tragweite des in Anspruch genommenen Grundrechts beruht, im Ergebnis zu einer unverhältnismäßigen Beschränkung der grundrechtlichen Freiheit führt oder willkürlich ist. ${ }^{158}$ Daher ist auch nur dieses Urteil selbst Gegenstand der Verfassungsbeschwerde. ${ }^{159}$ Für deren Erfolg muss das Urteil selbst - jenseits auch innerhalb des Verfassungsrechts zugestandener Rechtsanwendungs- und -auslegungsspielräume der Gerichte - gegen Grundrechte verstoßen. ${ }^{160}$

\section{Spezifische Grundrechtsverstöße}

Solche Grundrechtsverstöße lassen sich sowohl im Hinblick auf Art. 2 Abs. 1 i.V.m. Art. 1 Abs. 1 GG als auch im Hinblick auf Art. 3 Abs. 1 GG erkennen.

Zum einen hat sich herausgestellt, dass eine verlässliche Gefahrenprognose auf Grund bloßer „Zugehörigkeit“ zu einer gewalttätigen Gruppe, wie es im Falle des Beschwerdeführers geschah, gänzlich fehlschlägt.

Im Falle des Beschwerdeführers dienten allein die Vorgaben der SVRL als standardisierte Gefahrenprognose, ohne dass sich der Hausrechtsinhaber um eigene Aufklärung bemühte. Da sich, wie gezeigt, ein solches Vorgehen als ungeeignete Grundlage zur Ausübung des Hausrechts erwies, war auch diese Ausübung selbst ungeeignet, zukünftige Beeinträchtigungen seitens des Hausrechtsinhabers zu vermeiden. Damit muss die allgemeine Handlungsfreiheit des Hausrechtsinhabers gegenüber dem Allgemeinen Persönlichkeitsrecht des Beschwerdeführers im Wege praktischer Konkordanz zurücktreten. Folglich verkannte der BGH die dem Beschwerdeführer hier zu Gute kommende Tragweite von Art. 2 GG, ${ }^{161}$ sodass eine Verfassungsbeschwerde schon in diesem Punkt nicht aussichtslos erscheint.

Selbst wenn man aber eine Geeignetheit der getätigten Gefahrenprognose unterstellen möchte, so ist sie auf Grund der nach dem betroffenen Bundesligaspiel festgestellten Fakten jedenfalls derart vage, unbestimmt und nur auf eine Verwirklichungswahrscheinlichkeit von allenfalls wenigen Prozentpunkten zu reduzieren, ${ }^{162}$ dass sie, selbst wenn man ihre Richtigkeit zu Lasten des Beschwerdeführers unterstellen möchte, mit ihrer Konsequenz des Stadionverbots in einem groben Missverhältnis der entgegenstehenden Interessen zu Lasten wesentlicher Lebensbereiche des Klägers mündet. Auch angesichts dieser negativ ausfallenden Verhältnismäßigkeitsprü-

158 St. Rspr. des BVerfG nach der sog. „Heck'schen Formel“, vgl. nur Lechner/Zuck, a.a.O. (s.o. Rn. 157) m.w.N.

159 Vgl. Lechner/Zuck, a.a.O. (s.o. Fn. 157), \$90, Rn. 100.

160 Canaris, a.a.O. (s.o. Fn. 131), S. 28 f., bringt es prägnant auf den Punkt: Maßstab der Urteilsverfassungsbeschwerde ist, „ob die angegriffene Entscheidung von Verfassungs wegen ein anderes Ergebnis haben müsste" (Hervorh. im Original).

161 Vgl. Lechner/Zuck, a.a.O. (s.o. Fn. 157), $\$ 90$, Rn. 94.

162 Vgl. auch die instruktive Parallele zur Praxis der Kriminalprognose bei Klesczewski, a.a.O. (s.o. Fn. 5), S. 253, s. dazu bereits oben unter C. I. 4. B). 
fung ${ }^{163}$ kann ein weiterer Grundrechtsverstoß durch die BGH-Entscheidung festgestellt werden.

Gleiches gilt im Hinblick auf das Willkürverbot. Misst man den Sachverhalt der BGH-Entscheidung an den beschriebenen verfassungsrechtlichen Vorgaben, so folgt daraus, dass in diesem Fall das Stadionverbot gegen den Beschwerdeführer willkürlich festgesetzt wurde und sich auch die Fortsetzung staatlicher Willkür auf ihn auswirkte. Beide Verstöße erkannte der BGH dennoch für Recht und verstieß damit selbst gegen Art. 3 Abs. 1 GG. ${ }^{164}$

\section{Fehlerhafte Rechtsanwendung durch den $\mathrm{BGH}$}

Neben den spezifischen Grundrechtsverletzungen sei noch auf einen weiteren Fauxpas des BGH hingewiesen, der schon für sich allein genommen zum Erfolg einer Verfassungsbeschwerde führen kann. Der BGH geht anscheinend von dem possessorischen Unterlassungsanspruch als alleiniger Rechtsgrundlage für das Stadionverbot aus:

Da die Verhängung eines Hausverbots seine Grundlage in einem Unterlassungsanspruch nach $\int S 862$ Abs. 1 Satz 2, 1004 Abs. 1 Satz 2 BGB, hat, [...] $]^{165}$

Damit spricht er sich wohl ${ }^{166}$ gegen die von Breucker und Orth favorisierte Lösung aus, den Rechtsgrund in einem quasi-negatorischen Unterlassungsanspruch aus $\$ 1004$ Abs. 1 S. 2 i.V.m. $\$ 823$ Abs. 1 BGB zu sehen. ${ }^{167}$ Der Rekurs allein auf den possessorischen Unterlassungsanspruch hat jedoch zwangsläufig gem. $\mathbb{8} 864$ BGB eine zeitliche Beschränkung auf ein Jahr zur Folge. ${ }^{168}$ Ein hierauf gegründetes Stadionverbot müsste also spätestens nach Ablauf eines Jahres automatisch erlöschen. ${ }^{169}$ Dies geschah im vorliegenden Fall nicht; der BGH zog diese zwingende Rechtsfolge, welche hier zum 18. April 2007 hätte eintreten müssen, nicht einmal in Erwägung.

Allein schon wegen dieses offensichtlichen Verstoßes gegen geltendes Recht ist die BGH-Entscheidung als willkürlich einzustufen und folglich zu verwerfen, ${ }^{170}$ wenn es

163 Eine solche verlangt das BVerfG von den Instanzgerichten regelmäßig, Lechner/Zuck, a.a.O. (s.o. Fn. 157), $\mathbb{S} 90, \mathrm{Rn} .95$.

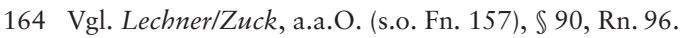

165 BGH, a.a.O. (s.o. Fn. 4), Rn. 16.

166 Es wird schon gar nicht deutlich, was diese Kette von Anspruchsgrundlagen eigentlich soll: Für den vom Besitzrecht unabhängigen possessorischen Anspruch ergibt sich der Unterlassungsanspruch unmittelbar aus $₫ 862$ Abs. 1 Satz 2 BGB. Des Rückgriffs auf $₫ 1004$ Abs. 1 Satz 2 bedarf es dafür nicht. $\$ 1004$ Abs. 2 Satz 1 BGB hilft in direkter Anwendung auch nicht recht weiter, weil der Verein (oder die Gesellschaft) auch im Profifußball regelmäßig nicht Stadioneigentümer ist.

167 Breucker, a.a.O. (s.o. Fn. 6), 134, 138; Orth, a.a.O. (s.o. Fn. 6), 137.

168 Überraschend moniert dies Breucker, SpuRt 2010, 31, nicht.

169 Breucker, a.a.O. (s.o. Fn. 6), 134; hierauf verweisend Orth, a.a.O. (s.o. Fn. 6), 137. Siehe bereits oben unter C. II. 6.

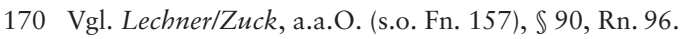


nicht gelingt, die verwendete Anspruchsgrundlage auszutauschen und durch eine tragfähige zu ersetzen. Denn jedenfalls seit dem 18. April 2007 verstieß das Stadionverbot offensichtlich und unauslegbar gegen $\$ 864$ BGB, weshalb dem Begehren des Klägers zumindest insoweit nach der vom BGH gewählten Anspruchsgrundlage hätte stattgegeben werden müssen. ${ }^{171}$

\section{E. Lösungsvorschlag: Ein vorläufiges Stadionverbot}

In der Praxis sind und bleiben damit diejenigen Stadionverbotsfälle unproblematisch, in denen der festsetzende Verein nicht nur bloße Kenntnis von der Einleitung eines Ermittlungsverfahrens, sondern darüber hinaus auch die ihm zu Grunde liegenden tatsächlichen Feststellungen erfährt. Aufgrund dieser Tatsachen kann er eine Gefahrprognose erstellen und, wenn diese tragfähig ist, ein bundesweites Stadionverbot aussprechen.

Problematisch bleiben die Fälle, in denen der ordnungspflichtige Verein lediglich von der Einleitung eines Ermittlungsverfahrens erfährt. Obwohl dies allein und für sich genommen die Verhängung eines jahrelangen Stadionverbots nicht rechtfertigen kann, kann danach - wenn willkürliches Handeln der Polizeibehörden ausgeschlossen ist - aufgrund des strafrechtlichen Anfangsverdachts ein Gefahrenverdacht dahin, dass der Betroffene an sicherheitsrelevanten Vorgängen beteiligt war und deswegen auch in Zukunft wieder beteiligt sein könnte, nicht ausgeschlossen werden. Dieser Gefahrenverdacht rechtfertigt natürlich kein Stadionverbot. Weil aber wegen der regelmäßig zeitnah anstehenden nächsten Ligaspiele ein schnelles und nachvollziehbares Schutzbedürfnis etwa im Hinblick auf die körperliche Unversehrtheit friedlicher Fußballfans besteht, kann dieser auch zivilrechtlich im Rahmen der aufgezeigten Anspruchsgrundlagen ein - angesichts des geringen Grades an Wiederholungsgefahr auf etwa zwei bis drei Wochen befristetes - vorläufiges bundesweites Stadionverbot rechtfertigen. Das allgemeine Interesse an einem gefährdungsfreien und reibungslosen Ablauf sämtlicher Profifußballspiele überwiegt in diesem Fall das Interesse eines möglicherweise zu Unrecht betroffenen Einzelnen, innerhalb des begrenzten Verbotszeitraums ein Stadion betreten zu dürfen. Bei der Mitteilung des vorläufigen Stadionverbots wird der Betroffene darauf hingewiesen, dass er zu den Vorwürfen angehört wird und zur Sache Stellung nehmen kann. Bestreitet der Betroffene seine Beteiligung nicht oder meldet er sich nicht zurück, ist seine sicherheitsrelevante Auffälligkeit im erheblichen Maße im zivilrechtlichen Sinne als zugestanden und unstreitig anzusehen und kann einer endgültigen Stadionverbotsentscheidung zu Grunde gelegt werden. Widerspricht der Betroffene, ist durch den aus-

171 Zugestanden sei allerdings, dass dies dem Begehren des Klägers, die Rechtswidrigkeit des Stadionverbots insgesamt und damit die Rückerlangung von Mitgliedschaft und Dauerkarte beim FC Bayern München, nicht unmittelbar gedient hätte. Seine Verfassungsbeschwerde müsste sich daher gegen das Urteil hinsichtlich der Feststellung der Rechtswidrigkeit des Stadionverbotssausspruchs dem Grunde nach und nicht lediglich der Dauer nach richten. 
sprechenden Verein eine dezidierte - und auch für den Betroffenen nachvollziehbare - schriftliche Gefahrprognose nach den vorstehend abgeleiteten Kriterien zu erstellen. Erfolgt dies nicht binnen einer angemessenen Frist von etwa bis zu weiteren drei Wochen, muss das vorläufige Stadionverbot aufgehoben werden.

Durch diesen Ansatz wäre nicht nur bessere Transparenz im Verfahrensgang gewährleistet, sondern es würden auch die betroffenen Fans aktiver in den Ablauf eingebunden. ${ }^{172}$ Beides kann die Akzeptanz des Mittels „Stadionverbot“ als solches deutlich steigern. Darüber hinaus trägt die Anhörung in erheblichem Umfang zur Sachverhaltsaufklärung bei und bietet die Möglichkeit, sich von dem Betroffenen ein individuelles Bild zu machen. ${ }^{173}$ Die persönliche Befragung des Betroffenen ist letztlich ein unerlässlicher Bestandteil einer validen Prognose über das zukünftige Verhalten eines Menschen. ${ }^{174}$

Hieraus folgt für die in der Praxis typischen Fälle einer Verfahrenseinstallung nach $\mathbb{} 153$ StPO, dass die Aufrechterhaltung eines Stadionverbots niemals grundsätzlich, sondern nur auf Grund einer Einzelfallprüfung nach den beschriebenen Maßstäben notwendig und rechtlich unbedenklich ist. Stellt die Staatsanwaltschaft aber das Ermittlungsverfahren nach $\mathbb{1 7 0}$ Abs. 2 StPO ein, ist wie nach der bereits bestehenden Rechtslage kein Stadionverbot möglich bzw. ist ein ergangenes ohne weitere Verfahrensschritte aufzuheben.

Hingegen kann die Rechtslage ${ }^{175}$ bei einer Einstellung nach $\$ 153$ a StPO verschärft werden: Mag auch mit ihr keine Schuldfeststellung verbunden sein, ${ }^{176}$ so verlangt $\$ 153$ a StPO doch eine gewisse Schuld- und Verurteilungswahrscheinlichkeit in der für die jeweilige Verfahrenslage vorgeschriebenen - also jedenfalls hinreichenden Verdachtsintensität. ${ }^{177}$ Zumindest diesen über einen bloßen Anfangsverdacht hinausgehenden Ermittlungsstand haben StA oder Gericht festgestellt und der Betroffene hat ihn - anders als im Fall von $\mathbb{\$} 153$ StPO - durch seine Zustimmung im Rahmen dieses freiwilligen Unterwerfungsverfahrens ${ }^{178}$ letztlich zugestanden. Die fakultative Aufhebungsmöglichkeit der SVRL kann für Härtefälle bestehen bleiben.

172 Die bislang untergeordnete Rolle der Anhörung beklagt auch zu Recht Klesczewski, a.a.O. (s.o. Fn. 5), S. 254.

173 Klesczewski, a.a.O. (s.o. Fn. 5), S. 254.

174 Klesczewski, a.a.O. (s.o. Fn. 5), S. 254 m.w.N.

175 S. oben zu $\$ 6$ Abs. 2 erster Spiegelstrich SVRL unter B. III. 2. b).

176 Löwe/Rosenberg-Beulke, 26. Aufl., 2008, $\$ 153$ a, Rn. 9; Meyer-Goßner, a.a.O., $\mathbb{} 153$ a, Rn. 10; BVerfG NJW 1991, 1530 ff.; für eine positive Schuldfeststellung hingegen KarlsruherKomm-Schoreit, a.a.O., $\$ 153$ a, Rn. 11 .

177 Löwe/Rosenberg-Beulke, a.a.O., Rn. 40.

178 Löwe/Rosenberg-Beulke, a.a.O., Rn. 10 m.w.N. 


\section{F. Schlussbemerkungen}

Fußballfans sind tatsächlich - abgesehen von den unverbesserlichen Absichtsstörern - keine Verbrecher, und das sollte man auch innerhalb so manch hitzig geführter Diskussion nicht vergessen. Ebenso wenig können Fußballfans nur wegen ihrer Faneigenschaft im Rahmen einer alltäglich anzutreffenden Gruppenzugehörigkeit pauschal als potenzielle Gefahrenquelle gleichsam inkriminiert werden. Daran ändert sich auch nichts, nur weil es immer ein paar wenige Unbelehrbare gibt, die sich aus dem Schutz der Gruppe heraus gewaltbereit zeigen. Letztere - und nur diese - gilt es systematisch und mit allen verfügbaren Mitteln zu verfolgen und dabei auch geeignete Präventivmaßnahmen zu treffen. Stadionverbote müssen sein für solche, die einfach nicht zu einem gewaltfreien Sport beitragen möchten. Alle Anderen genießen dagegen einen umfassenden grundrechtlichen Schutz gegen substanzlos begründete, willkürliche oder überzogene Stadionverbote. Der BGH wäre gut beraten gewesen, mit dem Reizthema „Stadionverbot“ differenzierter und einfühlsamer umzugehen. Dies fordert nicht nur unser Grundgesetz; eine solche Gangart könnte darüber hinaus zu einer bislang kaum zu verzeichnenden Akzeptanz des vom Grunde her wirksamen und sinnvollen Mittels „Stadionverbot“ unter den Fans verhelfen. Deswegen sollte es im Interesse der Fußballverbände liegen, das Stadionverbot auf rechtlich und rechtsstaatlich unangreifbare Grundlagen zu stellen. 